\title{
Dilations of Some VH-Spaces Operator Valued Invariant Kernels
}

\author{
Aurelian Gheondea
}

\begin{abstract}
We investigate VH-spaces (Vector Hilbert spaces, or Loynes spaces) operator valued Hermitian kernels that are invariant under actions of $*$-semigroups from the point of view of generation of $*$-representations, linearizations (Kolmogorov decompositions), and reproducing kernel spaces. We obtain a general dilation theorem in both Kolmogorov and reproducing kernel space representations, that unifies many dilation results, in particular B. Sz.-Nagy's and Stinesprings' dilation type theorems.
\end{abstract}

Mathematics Subject Classification (2010). Primary 47A20;

Secondary 43A35, 46E22, 46L89.

Keywords. VH-space, admissible space, Hermitian kernel, positive semidefinite kernel, invariant kernel, reproducing kernel, Kolmogorov decomposition, completely positive map, *-semigroup, *-representation, $B^{*}$-algebra, dilation.

\section{Introduction}

The dilation theorem of Sz.-Nagy [31], which generalizes a dilation theorem for groups of Naimark [25], says that any operator valued positive semidefinite map on a $*$-semigroup can be dilated to a $*$-representation of the $*$-semigroup on a "larger" Hilbert space. A generalization to VH-spaces (Vector Hilbert spaces) operator valued maps, motivated by questions in multivariable stochastic processes, was obtained by Loynes [17]. A slightly stronger version of this generalization was obtained in [11].

The Stinespring's Theorem [29], which generalizes another dilation theorem, for semispectral measures, of Naimark [24], says that, for the case of a Hilbert space $\mathcal{H}$ and a $C^{*}$-algebra $\mathcal{A}$, any positive semidefinite map $\varphi: \mathcal{A} \rightarrow \mathcal{B}(\mathcal{H})$ can be dilated to a $*$-homomorphism $\pi$ of $\mathcal{A}$ on $\mathcal{B}(\mathcal{K})$, for

This work was supported by a grant of the Romanian National Authority for Scientific Research, CNCS UEFISCDI, project number PN-II-ID-PCE-2011-3-0119. 
some "larger" Hilbert space $\mathcal{K}$. A result of Szafraniec [30] says that Stinespring's Theorem is logically equivalent with the Sz.-Nagy Dilation Theorem. An enhanced version of Stinespring's Theorem, e.g. see [3], states that Stinespring's Theorem is also true when $\mathcal{A}$ is a $B^{*}$-algebra. It was proven in [11] that a variant of this equivalence can be put in the framework of VH-spaces, that is, the theorem of Szafraniec extends to the setting of VH-spaces as well.

The aim of this article is to show that these two dilation theorems, even at the level of generality of VH-spaces operator valued maps, can be unified under the same concept, those of positive semidefinite kernels that are invariant under the action of a $*$-semigroup. More precisely, we prove in Theorem 4.2 that these kernels can be equivalently characterized by a "linearization" of the kernel, that is called a Kolmogorov decomposition, together with a $*$-representation of the $*$-semigroup onto a "larger" VH-space, and we also show that, even more, this is equivalent with a VH-space reproducing kernel onto which the $*$-representation holds

Positive semidefinite kernels have been first considered mainly with respect to reproducing kernel Hilbert spaces, see Aronszajn [1] and the rich bibliography cited there. An equivalent description can be obtained by Kolmogorov decomposition, which is a linearization (or separation of variables) of the kernel, named this way after Kolmogorov's seminal paper [15]. For scalar valued kernels, this linearization was first obtained by J. Mercer back in 1909, cf. [21], in connection with the theory of integral equations as developed by D. Hilbert, while the reproducing kernel aspects have been systematically considered by Moore [22]. Basically, a Kolmogorov decomposition is a dilation phenomenon that is strongly related with many other problems in operator algebras and mathematical physics, see Parthasarathy and Schmidt [26], Evans and Lewis [7] and the rich bibliography cited there. When the kernel presents a certain symmetry, that can be modeled, e.g. by an invariance with respect to an action of a *-semigroup (any group can be organized in a natural way as a *-semigroup with the involution defined by the inverse operation), this turns out to be a powerful method of producing representations of the underlying *-semigroup on the Hilbert space of dilation. A consequence is that this unifies both Sz.-Nagy's type dilations and Stinespring's type dilations. These ideas have been used in investigating dilations for indefinite Hermitian kernels in [4,5].

Motivated by questions in operator algebras and mathematical physics, some generalizations of Hilbert spaces to the case when the inner product takes vector values have been investigated: we mention here Hilbert modules, cf. the monographs $[16,20]$, notably a generalization to Hilbert $C^{*}$-module operator valued maps of the Stinespring's Theorem obtained by Kasparov [14], Hilbert modules over locally $C^{*}$-algebras, cf. Inoue [13] and Phillips [28], as well as a different type generalization, that was performed by R.M. Loynes, notably his generalization of the Sz.-Nagy's dilation theorem as in [17], and followed by a study of operators on these spaces, as in [18]. The latter vector valued Hilbert spaces, that have been acronymed by VH-spaces, show many common features with Hilbert spaces but there are many anomalies as well, the most notable ones due to missing a Schwarz Inequality and an 
analog of the Riesz's Representation Theorem. Motivation for studying these $\mathrm{VH}$-spaces and their linear operators originally came mainly from multi-variable stochastic processes, as explained in [19], see also [8] and the rich bibliography cited there for applications of this theory and for an update review of these applications.

It is worth noting that $\mathrm{VH}$-spaces are so general that they contain Hilbert modules over either $C^{*}$ or locally $C^{*}$-algebras. From this perspective, more recently, Murphy [23] considered Kolmogorov decompositions in connection with Hilbert $C^{*}$-modules, Gaşpar and Gaşpar studied reproducing kernel Hilbert $B(X)$-modules in [10] and reproducing kernel Hilbert modules over locally $C^{*}$-algebras in [9], while Heo [12] investigated reproducing kernel Hilbert $C^{*}$-modules and kernels associated with cocycles.

We start this article with a brief presentation of notation and basic facts on VH-spaces, their linear operators and the $C^{*}$-algebra of adjointable operators, to which we add an inequality related to tensor products of Hilbert spaces with VH-spaces, as a technical result needed later in this article. Then we consider Hermitian kernels that take values in $\mathcal{B}^{*}(\mathcal{H})$, the $C^{*}$ algebra of adjointable operators on a $\mathrm{VH}$-space $\mathcal{H}$ and investigate different levels of positivity and their consequences. There are two main results here: one is Theorem 3.3 that shows that Kolmogorov decompositions characterize positive semidefinte kernels and the second is Theorem 3.5 that adds the characterization by reproducing kernel $\mathrm{VH}$-spaces. There are advantages and disadvantages for each one of these: Kolmogorov decomposition gives much more freedom in dealing with it, while its reproducing kernel counter-part has a "function space" look and enjoys uniqueness. In view of our experience with applications of the operator valued kernels to moment problems, dilations theory, and multi-variable holomorphy as in [6], we think that having both of them available is an advantage on the flexibility side, which offers a choice depending on the particular problem that requires this model.

The main result is Theorem 4.2 that shows that, when the kernel is invariant under the action of a certain $*$-semigroup, then the Kolmogorov decomposition, as well as its underlying reproducing kernel VH-space, yields a $*$-representation of the $*$-semigroup on the VH-space of dilation, that can be viewed also on the underlying reproducing kernel VH-space. Then we show that the Loynes-Sz.-Nagy dilation type theorem, see Theorem 5.2 obtained in [11], is a particular case of Theorem 4.2 to which we add an equivalent characterization in terms of reproducing kernels. In addition, we transfer Kolmogorov decompositions to linearizations of positive semidefinite maps on $*$-semigroups. Finally, we show that the Stinespring's type theorem for $\mathrm{VH}$-spaces operator valued completely positive maps on $B^{*}$-algebras obtained in [11], see Theorem 5.5, can be obtained from Thereom 4.2 as well.

\section{Notation and Preliminary Results on VH-Spaces}

In this section we review most of the definitions and some theorems on VH-spaces and their operator theory, cf. Loynes [17-19]. A few facts are 
added in connection to taking tensor products, in order to obtain a technical result that can be considered as a surrogate of a multivariable Schwarz inequality.

\subsection{VH-Spaces}

A complex vector space $Z$ is called admissible if:

(a1) $Z$ is a complete locally convex space.

(a2) $Z$ has an involution $*$, that is, a map $Z \ni z \mapsto z^{*} \in Z$ that is conjugate linear $\left((\alpha x+\beta y)^{*}=\bar{\alpha} x^{*}+\bar{\beta} y^{*}\right.$ for all $\alpha, \beta \in \mathbb{C}$ and all $\left.x, y \in Z\right)$ and involutive $\left(\left(z^{*}\right)^{*}=z\right.$ for all $\left.z \in Z\right)$.

(a3) In $Z$ there is a convex cone $Z_{+}\left(\alpha x+\beta y \in Z_{+}\right.$for all numbers $\alpha, \beta \geq 0$ and all $\left.x, y \in Z_{+}\right)$, that is closed, strict $\left(Z_{+} \cap-Z_{+}=\{0\}\right)$, and consisting of selfadjoint elements only $\left(z^{*}=z\right.$ for all $\left.z \in Z_{+}\right)$. This cone is used to define a partial order in $Z$ by: $z_{1} \geq z_{2}$ if $z_{1}-z_{2} \in Z_{+}$.

(a4) The topology of $Z$ is compatible with the partial ordering in the sense there exists a base of the topology, linearly generated by a family of neighbourhoods $\left\{N_{j}\right\}_{j \in \mathcal{J}}$ of the origin, such that all of them are convex and solid, that is, whenever $x \in N_{j}$ and $0 \leq y \leq x$ then $y \in N_{j}$.

It can be proven that axiom (a4) is equivalent with the following one:

(a4') There exists a collection of seminorms $\left\{p_{j}\right\}_{j \in \mathcal{J}}$ defining the topology of $Z$ that are increasing, that is, $0 \leq x \leq y$ implies $p_{j}(x) \leq p_{j}(y)$.

If, in addition, to the axioms (a1)-(a4), the space $Z$ satisfies also the following:

(a5) With respect to the specified partial ordering, any bounded monotone sequence is convergent.

then $Z$ is called a strongly admissible space.

Given a complex linear space $\mathcal{E}$ and an admissible space $Z$, a $Z$-valued inner product or $Z$-gramian is, by definition, a mapping $\mathcal{E} \times \mathcal{E} \ni(x, y) \mapsto$ $[x, y] \in Z$ subject to the following properties:

(ve1) $[x, x] \geq 0$ for all $x \in \mathcal{E}$, and $[x, x]=0$ if and only if $x=0$.

(ve2) $[x, y]=[y, x]^{*}$ for all $x, y \in \mathcal{E}$.

(ve3) $\left[a x_{1}+b x_{2}, y\right]=a\left[x_{1}, y\right]+b\left[x_{2}, y\right]$ for all $a, b \in \mathbb{C}$ and all $x_{1}, x_{2} \in \mathcal{E}$.

A complex linear space $\mathcal{E}$ onto which a $Z$-valued inner product $[\cdot, \cdot]$ is specified, for a certain admissible space $Z$, is called a $V E$-space (Vector Euclidean space).

In any VE-space $\mathcal{E}$ over an admissible space $Z$ the familiar polarization formula

$$
4[x, y]=\sum_{k=0}^{3} \mathrm{i}^{k}\left[\left(x+\mathrm{i}^{k} y, x+\mathrm{i}^{k} y\right], \quad x, y \in \mathcal{E},\right.
$$

holds, which shows that the $Z$-valued inner product is perfectly defined by the $Z$-valued quadratic form $\mathcal{E} \ni x \mapsto[x, x]$.

Any VE-space $\mathcal{E}$ can be made in a natural way into a Hausdorff separated locally convex space by considering the weakest locally convex topology on $\mathcal{E}$ that makes the mapping $E \ni h \mapsto[h, h] \in Z$ continuous, more precisely, 
letting $\left\{N_{j}\right\}_{j \in \mathcal{J}}$ be the collection of convex and solid neighbourhoods of the origin in $Z$ as in axiom (a4), the collection of sets

$$
U_{j}=\left\{x \in \mathcal{E} \mid[x, x] \in N_{j}\right\}, \quad j \in \mathcal{J},
$$

is a topological base of neighbourhoods of the origin of $\mathcal{E}$ that linearly generates the weakest locally convex topology on $\mathcal{E}$ that makes the mapping $\mathcal{E} \ni h \mapsto[h, h] \in Z$ continuous, cf. Theorem 1 in [17]. In terms of seminorms, this topology can be defined in the following way: let $\left\{p_{j}\right\}_{j \in \mathcal{J}}$ be a family of increasing seminorms defining the topology of $Z$ and let

$$
q_{j}(h)=p_{j}([h, h])^{1 / 2}, \quad h \in \mathcal{E}, j \in \mathcal{J} .
$$

Then the specified topology of $\mathcal{E}$ is fully determined by the family of seminorms $\left\{q_{j}\right\}_{j \in \mathcal{J}}$.

If $\mathcal{E}$ is complete with respect to this locally convex topology then it is called a $V H$-space (Vector Hilbert space). In case the admissible space $Z$ is strongly admissible, a VH-space is called an $L V H$-space (Limit Vector Hilbert space), cf. [18], or Loynes space. LVH-spaces are more suitable for spectral representations of their unitary or selfadjoint operators, but we do not use them in this article.

The concept of VE-spaces isomorphism is also naturally defined: this is just a linear bijection $U: \mathcal{E} \rightarrow \mathcal{F}$, for two VE-spaces over the same admissible space $Z$, such that, $[U x, U y]=[x, y]$ for all $x, y \in \mathcal{E}$. Any VE-space $\mathcal{E}$ can be embedded as a dense subspace of a $\mathrm{VH}$-space $\mathcal{H}$, uniquely determined up to an isomorphism, cf. Theorem 2 in [17].

In general VH-spaces, an analog of the Schwarz Inequality does not hold. However, some of its consequences can be proven using slightly different techniques. One such consequence is the following lemma.

Lemma 2.1. (Loynes [17]) Let $Z$ be an admissible space, $\mathcal{E}$ a complex vector space and $[\cdot, \cdot]: \mathcal{E} \times \mathcal{E} \rightarrow Z$ a positive semidefinite sesqui-linear map, that is, $[\cdot, \cdot]$ is linear in the first variable and conjugate linear in the second variable, and $[x, x] \geq 0$ for all $x \in \mathcal{E}$. If $f \in \mathcal{E}$ is such that $[f, f]=0$, then $\left[f, f^{\prime}\right]=\left[f^{\prime}, f\right]=0$ for all $f^{\prime} \in \mathcal{E}$.

\subsection{Linear Operators in VH-Spaces}

The collection $\mathcal{L}(\mathcal{E}, \mathcal{F})$ of all linear and continuous operators between VE-spaces $\mathcal{E}$ and $\mathcal{F}$ is naturally organized as a complex vector space. In particular, the set $\mathcal{L}(\mathcal{E})$ of all linear and continuous operators $T: \mathcal{E} \rightarrow \mathcal{E}$ is naturally organized as a complex algebra.

Given two VH-spaces $\mathcal{H}$ and $\mathcal{K}$, a linear operator $A: \mathcal{H} \rightarrow \mathcal{K}$ is called bounded if there exists a constant $k \geq 0$ such that

$$
[A x, A x] \leq k[x, x], \quad x \in \mathcal{H} .
$$

Taking into account the definition of the underlying topology of a VH-space, any linear and bounded operator $T$ is continuous but the converse is not true, in general. We denote the special class of bounded operators by $\mathcal{B}(\mathcal{H}, \mathcal{K})$. For a bounded operator $A \in \mathcal{B}(\mathcal{H}, \mathcal{K})$ we define its operator norm $\|A\|$ by the 
square root of the least $k$ satisfying (2.4), that is,

$$
\|A\|=\inf \{\sqrt{k} \mid[A x, A x] \leq k[x, x], \text { for all } x \in \mathcal{H}\} .
$$

It is easy to see that the infimum is actually a minimum and hence, that we have

$$
[A x, A x] \leq\|A\|^{2}[x, x], \quad x \in \mathcal{H} .
$$

If $\mathcal{H}$ is a VH-space then $\mathcal{B}(H)=\mathcal{B}(H, H)$ is a Banach algebra with respect to the usual algebraic operations and the operator norm, cf. Theorem 1 in [18].

\subsection{The $C^{*}$-Algebra $\mathcal{B}^{*}(\mathcal{H})$}

Given two VH-spaces $\mathcal{H}$ and $\mathcal{K}$, an operator $A \in \mathcal{B}(\mathcal{H}, \mathcal{K})$ is called adjointable if there exists a bounded operator $A^{*}: \mathcal{K} \rightarrow \mathcal{H}$ such that for all $x \in \mathcal{H}$ and $y \in \mathcal{K}$

$$
[A x, y]=\left[x, A^{*} y\right] .
$$

We denote by $\mathcal{B}^{*}(\mathcal{H}, \mathcal{K})$ the collection of all adjointable elements in $\mathcal{B}(\mathcal{H}, \mathcal{K})$. We emphasize the fact that, in a general VH-space setting, not all bounded operators are adjointable. This is mostly due to the lack of an analog of the Riesz Representation Theorem.

The definitions of selfadjoint, unitary, and normal operators are the same as in the Hilbert space case. It is clear that $A$ is selfadjoint if and only if $[A x, y]=[x, A y]$ for all $x, y \in \mathcal{H}$, and also, by the polarization formula (2.1), this is equivalent to

$$
[A x, x]=[A x, x]^{*}, \quad x \in \mathcal{H} .
$$

A bounded operator $A$ in $\mathcal{H}$ is called positive if $[A x, x] \geq 0$ for all $x \in \mathcal{H}$. From (2.8) it follows that a positive operator is necessarily selfadjoint. A contraction is a linear operator $T$ such that $[T x, T x] \leq[x, x]$ for all $x \in \mathcal{H}$. By Theorem 2 in [18], the involution $*$ is isometric, that is, $\left\|T^{*}\right\|=\|T\|$.

If $A \in \mathcal{B}^{*}(\mathcal{H})$ is selfadjoint, then we have

$$
\text { - }\|A\|[x, x] \leq[A x, x] \leq\|A\|[x, x] .
$$

The importance of the previous inequality, cf. Theorem 3 in [18], is that, sometimes, it may be used instead of the Schwarz Inequality which, in general, does not hold for a VH-space. Moreover, assume that $A$ is a linear operator in $\mathcal{H}$ and that for some real numbers $m, M$ we have

$$
m[x, x] \leq[A x, x] \leq M[x, x], \quad x \in \mathcal{H} .
$$

Then $A \in \mathcal{B}^{*}(\mathcal{H})$ and $A=A^{*}$. If, in addition, $m$ is the maximum and $M$ is the minimum with these properties, then $|A|=\max \{|m|,|M|\}$.

It is now clear that $\mathcal{B}^{*}(\mathcal{H})$ is a Banach $*$-algebra with isometric involution. According to Theorem 4 in [18], for any VH-space $\mathcal{H}$ the algebra $\mathcal{B}^{*}(\mathcal{H})$ is a $C^{*}$-algebra, more precisely, we have $\left\|A^{*} A\right\|=\|A\|^{2}$ for all $A \in \mathcal{B}^{*}(\mathcal{H})$.

On the other hand, the natural cone of positive elements in a $C^{*}$-algebra $\mathcal{A}$ is $\mathcal{A}^{+}=\left\{a^{*} a \mid a \in \mathcal{A}\right\}$. According to Theorem 5 in [18], given $\mathcal{H}$ a VH-space and $A \in \mathcal{B}^{*}(\mathcal{H})$, then $A$ is positive (that is, $[A x, x] \geq 0$ for all 
$x \in \mathcal{H})$ if and only if $A=B^{*} B$ for some $B \in \mathcal{B}^{*}(\mathcal{H})$. So, the two notions coincide.

A subspace $\mathcal{M}$ of a $\mathrm{VH}$-space $\mathcal{H}$ is orthocomplemented or accessible if every element $x \in \mathcal{H}$ can be written as $x=y+z$ where $y$ is in $\mathcal{M}$ and $z$ is such that $[z, m]=0$ for all $m \in \mathcal{M}$, that is, $z$ is in the orthogonal companion $\mathcal{M}^{\perp}$ of $\mathcal{M}$. Observe that if such a decomposition exists it is unique and hence the orthogonal projection $P_{\mathcal{M}}$ onto $\mathcal{M}$ can be defined by $P_{\mathcal{M}} x=y$. Any orthogonal projection $P$ is selfadjoint and idempotent, in particular we have $[P x, y]=[P x, P y]$ for all $x, y \in \mathcal{H}$, hence $P$ is positive and contractive. Conversely, any selfadjoint idempotent operator is an orthogonal projection onto its range subspace. Any orthocomplemented subspace is closed.

\subsection{Direct Sums and Tensor Products}

Let $Z$ be an admissible space and $\mathcal{H}_{k}, k=1, \ldots, n$ be VH-spaces with $Z$-gramian $[\cdot, \cdot]_{k}$, respectively. On the algebraic direct sum $\mathcal{K}=\mathcal{H}_{1} \oplus \cdots \oplus \mathcal{H}_{n}$ one can consider a two variable $Z$-valued map

$$
[x, y]=\sum_{k=1}^{n}\left[x_{k}, y_{k}\right]_{k}, \quad x=\left(x_{1}, \ldots, x_{n}\right), y=\left(y_{1}, \ldots, y_{n}\right),
$$

and it is easy to see that it is a $Z$-valued inner product on $\mathcal{H}$. Letting $\left\{p_{j}\right\}_{j}$ denote a collection of increasing seminorms on $Z$ that define its underlying topology, we consider the family of seminorms on $\mathcal{K}$

$$
\mathcal{K} \ni x \mapsto\left(\sum_{k=1}^{n} p_{j}\left(\left[x_{k}, x_{k}\right]_{k}\right)\right)^{1 / 2}, \quad x=\left(x_{1}, \ldots, x_{n}\right),
$$

and it is easy to see that the locally convex topology of $\mathcal{K}$ defined by this family of seminorms is complete, hence $\mathcal{K}$ is a VH-space.

If we have $\mathcal{H}_{k}=\mathcal{H}$ for all $k=1, \ldots, n$ then we let $\mathcal{H}^{n}=\mathcal{H} \oplus \cdots \oplus \mathcal{H}$ (the sum has exactly $n$ terms). An alternate characterization of the VH-space $\mathcal{H}^{n}$ can be obtained as a tensor product. More precisely, let $\mathbb{C}^{n}$ denote the canonical $n$-dimensional complex vector space and consider the algebraic tensor product $\mathbb{C}^{n} \otimes \mathcal{H}$, on which a $Z$ - valued two variable map can be defined by

$$
\left[\sum_{k=1}^{l} e_{k} \otimes x_{k}, \sum_{j=1}^{m} f_{j} \otimes y_{j}\right]=\sum_{k=1}^{l} \sum_{j=1}^{m}\left\langle e_{k}, f_{j}\right\rangle\left[x_{k}, y_{j}\right],
$$

for $x_{k}, y_{j} \in \mathbb{C}^{n}$ and $e_{k}, f_{j} \in \mathcal{H}, k=1, \ldots, l$ and $j=1, \ldots, m$. By Proposition 2.4 in [11], given $\mathcal{H}$ a VH-space and $n \in \mathbb{N}$, the vector space $\mathbb{C}^{n} \otimes \mathcal{H}$, endowed with the $Z$-valued map $[\cdot, \cdot]$ defined by $(2.11)$, is a VH-space, canonically isomorphic with the $\mathrm{VH}$-space $\mathcal{H}^{n}$.

Let $M_{n}$ denote the $C^{*}$-algebra of all $n \times n$ matrices with complex entries. There is a canonical identification of $M_{n}$ with the $C^{*}$-algebra $\mathcal{B}\left(\mathbb{C}^{n}\right)$ given by the action on the canonical orthonormal basis of $\mathbb{C}^{n}$. We consider $M_{n}\left(\mathcal{B}^{*}(\mathcal{H})\right)$ as the collection of all $n \times n$ matrices with entries in $\mathcal{B}^{*}(\mathcal{H})$ that has a natural structure of $*$-algebra: for instance, letting $A=\left[A_{i, j}\right]_{i, j=1}^{n}$ we have $A^{*}=\left[A_{j, i}^{*}\right]_{i, j=1}^{n}$, addition is entry-wise, while multiplication is matrix-wise. 
Since $\mathcal{B}^{*}(\mathcal{H})$ is a $C^{*}$-algebra, $M_{n}\left(\mathcal{B}^{*}(\mathcal{H})\right)$ is a $C^{*}$-algebra, e.g. see [27], in a natural fashion. There is a canonical identification of $M_{n}\left(\mathcal{B}^{*}(\mathcal{H})\right)$ with the $C^{*}$-algebra $\mathcal{B}^{*}\left(\mathcal{H}^{n}\right)$ which provides a $*$-isomorphism of $C^{*}$-algebras, more precisely, any $A=\left[A_{i, j}\right]_{i, j=1}^{n}$ in $M_{n}\left(\mathcal{B}^{*}(\mathcal{H})\right)$ is identified with the operator $A$ in $\mathcal{H}^{n}$ defined by left matrix multiplication with column vectors of size $n$ with entries in $\mathcal{H}$.

Consider now the vector space $M_{n} \otimes \mathcal{B}^{*}(\mathcal{H})$. There is a natural structure of $*$-algebra on $M_{n} \otimes \mathcal{B}^{*}(\mathcal{H})$ : for elementary tensors $A \otimes T$ and $B \otimes S$, we have

$$
(A \otimes T)(B \otimes S)=(A B \otimes T S), \quad(A \otimes T)^{*}=A^{*} \otimes T^{*} .
$$

Moreover, an identification of the $*$-algebra $M_{n} \otimes \mathcal{B}^{*}(\mathcal{H})$ with the $C^{*}$-algebra $M_{n}\left(\mathcal{B}^{*}(\mathcal{H})\right)$ is obtained in the following way: for an elementary tensor $A \otimes T$ the corresponding element in $M_{n}\left(\mathcal{B}^{*}(\mathcal{H})\right)$ is $\left[a_{i, j} T\right]_{i, j=1}^{n}$. This provides a natural $C^{*}$-algebra structure on $M_{n} \otimes \mathcal{B}^{*}(\mathcal{H})$ with respect to which this identification becomes a $*$-isomorphism.

On the other hand, since the $C^{*}$-algebras $M_{n}$ and $\mathcal{B}\left(\mathbb{C}^{n}\right)$ are identified canonically, we actually have a canonical identification of the $C^{*}$-algebras $M_{n} \otimes \mathcal{B}^{*}(\mathcal{H})$ with the $C^{*}$-algebra $\mathcal{B}^{*}\left(\mathbb{C}^{n} \otimes \mathcal{H}\right)$ : an arbitrary elementary tensor $A \otimes T$ in $M_{n} \otimes \mathcal{B}^{*}(\mathcal{H})$ is identified with the operator on the VH-space $\mathbb{C}^{n} \otimes \mathcal{H}$ by

$$
(A \otimes T)(x \otimes h), \quad x \in \mathbb{C}^{n}, h \in \mathcal{H},
$$

and then extended by linearity. We are particularly interested in positive elementary tensors: if $A \in M_{n}^{+}$and $T \in \mathcal{B}^{*}(\mathcal{H})^{+}$, then $A \otimes T \in\left(M_{n} \otimes \mathcal{B}^{*}(\mathcal{H})\right)^{+}$, more precisely, if $A=B^{*} B$ for some $B \in M_{n}$ and similarly, $T=S^{*} S$ for some $S \in \mathcal{B}^{*}(\mathcal{H})$, hence, $A \otimes T=B^{*} B \otimes S^{*} S=\left(B^{*} \otimes S^{*}\right)(B \otimes S)=(B \otimes S)^{*}(B \otimes S)$.

The following inequality is a surrogate of a Schwarz inequality and will be needed later.

Lemma 2.2. Let $T$ be a positive operator in the $V H$-space $\mathcal{H}$. Then, for all $h_{1}, h_{2}, \ldots, h_{n} \in \mathcal{H}$ we have

$$
0 \leq \sum_{j, k=1}^{n}\left[T h_{j}, h_{k}\right]_{\mathcal{H}} \leq\|T\| \sum_{j, k=1}^{n}\left[h_{j}, h_{k}\right]_{\mathcal{H}} .
$$

Proof. We consider the VH-space $\mathcal{H}^{n}=\mathbb{C}^{n} \otimes \mathcal{H}$ and then the $C^{*}$-algebra

$$
M_{n}\left(\mathcal{B}^{*}(\mathcal{H})\right) \simeq M_{n} \otimes \mathcal{B}^{*}(\mathcal{H}) \simeq \mathcal{B}^{*}\left(\mathcal{H}^{n}\right) .
$$

We consider $E \in M_{n}$ the $n \times n$ matrix with all entries equal to 1 and note that it is positive. Since $T$ is positive it follows that $\|T\| I-T \geq 0$ and hence

$$
E \otimes(\|T\| I)-E \otimes T=E \otimes(\|T\| I-T) \geq 0,
$$

as an element in the $C^{*}$-algebra $M_{n} \otimes \mathcal{B}^{*}(\mathcal{H})$ as before, equivalently,

$$
E \otimes T \leq E \otimes(\|T\| I)=\|T\|(E \otimes I),
$$

which, when evaluated at the vector $\left(h_{1}, h_{2}, \ldots, h_{n}\right) \in \mathcal{H}^{n} \simeq \mathbb{C}^{n} \otimes \mathcal{H}$, provides the inequality (2.13). 


\section{Hermitian Kernels}

\subsection{Some General Facts}

Let $X$ be a nonempty set and let $\mathcal{H}$ be a VH-space over the admissible space $Z$. A map $K: X \times X \rightarrow \mathcal{B}(\mathcal{H})$ is called a kernel on $X$ and valued in $\mathcal{B}(\mathcal{H})$. In case the kernel $K$ has values in $\mathcal{B}^{*}(\mathcal{H})$, an adjoint kernel $K^{*}: X \times X \rightarrow \mathcal{B}^{*}(\mathcal{H})$ can be associated by $K^{*}(x, y)=K(y, x)^{*}$ for all $x, y \in X$. The kernel $K$ is called Hermitian if $K^{*}=K$.

Let $\mathcal{F}=\mathcal{F}(X ; \mathcal{H})$ denote the complex vector space of all functions $f: X \rightarrow \mathcal{H}$, and $\mathcal{G}=\mathcal{G}(X ; \mathcal{H})$ its subspace of those functions having finite support. A pairing $[\cdot, \cdot]_{\mathcal{G}}: \mathcal{G} \times \mathcal{G} \rightarrow Z$ can be defined by

$$
[g, h]_{\mathcal{G}}=\sum_{y \in X}[g(y), h(y)]_{\mathcal{H}}, \quad g, h \in \mathcal{G} .
$$

This pairing is clearly a $Z$-gramain on $\mathcal{G}$, hence $\left(\mathcal{G} ;[\cdot, \cdot]_{\mathcal{G}}\right)$ is a VE-space.

Let us observe that the sum in (3.1) makes sense even when only one of the functions $g$ or $h$ has finite support, the other can be arbitrary in $\mathcal{F}$. Thus, another pairing $[\cdot, \cdot]_{K}$ can be defined on $\mathcal{G}$ by

$$
[g, h]_{K}=\sum_{x, y \in X}[K(y, x) g(x), h(y)]_{\mathcal{H}}, \quad g, h \in \mathcal{G} .
$$

In general, the pairing $[\cdot, \cdot]_{K}$ is linear in the first variable and conjugate linear in the second variable. If, in addition, $K=K^{*}$ then the pairing $[\cdot, \cdot]_{K}$ is Hermitian as well, that is,

$$
[g, h]_{K}=[h, g]_{K}^{*}, \quad g, h \in \mathcal{G} .
$$

A convolution operator $K: \mathcal{G} \rightarrow \mathcal{F}$ can be associated to the kernel $K$ by

$$
(K g)(y)=\sum_{x \in X} K(y, x) g(x), \quad g \in \mathcal{G},
$$

and it is easy to see that $K$ is a linear operator. There is a natural relation between the pairing $[\cdot, \cdot]_{K}$ and the convolution operator $K$ given by

$$
[g, h]_{K}=[K g, h]_{\mathcal{G}}, \quad g, h \in \mathcal{G} .
$$

If $K$ is adjointable, and letting $K^{*}$ denote the convolution operator of the adjoint kernel $K^{*}$, we have

$$
[g, h]_{K}=[K g, h]_{\mathcal{G}}=\left[g, K^{*} h\right]_{\mathcal{G}}=\left[K^{*} h, g\right]_{\mathcal{G}}^{*}=[h, g]_{K^{*}}^{*}, \quad g, h \in \mathcal{G},
$$

and hence, the pairing $[\cdot, \cdot]_{K}$ is Hermitian if and only the kernel $K$ is Hermitian.

Given $n \in \mathbb{N}$, the kernel $K$ is called $n$-positive if for any $x_{1}, x_{2}, \ldots, x_{n} \in$ $X$ and any $h_{1}, h_{2}, \ldots, h_{n} \in \mathcal{H}$ we have

$$
\sum_{j, k=1}^{n}\left[K\left(x_{k}, x_{j}\right) h_{j}, h_{k}\right]_{\mathcal{H}} \geq 0 .
$$

The kernel $K$ is called positive semidefinite (or of positive type) if it is $n$-positive for all natural numbers $n$. 
Lemma 3.1. Assume that the kernel $K: X \times X \rightarrow \mathcal{B}^{*}(\mathcal{H})$ is 2-positive. Then: (1) $K$ is Hermitian.

(2) If, for some $x \in X$, we have $K(x, x)=0$, then $K(x, y)=0$ for all $y \in X$.

Proof. (1) Since $K$ is 2-positive it is 1-positive, hence $K(x, x) \geq 0$ for all $x \in X$. On the other hand, writing down the 2-positivity condition, for any $x, y \in X$ and any $g, h \in \mathcal{H}$ we have

$$
[K(x, y) g, h]_{\mathcal{H}}+[K(y, x) h, g]_{\mathcal{H}}+[K(x, x) h, h]_{\mathcal{H}}+[K(y, y) g, g]_{\mathcal{H}} \geq 0,
$$

hence the sum of the first two terms is in the real span of the cone $Z_{+}$, in particular, it is selfadjoint. Thus,

$$
[K(x, y) g, h]_{\mathcal{H}}+[K(y, x) h, g]_{\mathcal{H}}=[h, K(x, y) g]_{\mathcal{H}}+[g, K(y, x) h]_{\mathcal{H}},
$$

equivalently,

$$
\left[\left(K(x, y)-K(y, x)^{*}\right) g, h\right]_{\mathcal{H}}+\left[\left(K(y, x)-K(x, y)^{*}\right) h, g\right]_{\mathcal{H}}=0 .
$$

Letting $h=\mathrm{i}\left(K(x, y)-K(y, x)^{*}\right) g$ it follows

$$
2 \mathrm{i}\left[\left(K(x, y)-K(y, x)^{*}\right) g,\left(K(x, y)-K(y, x)^{*}\right) g\right]_{\mathcal{H}}=0,
$$

hence $K(x, y)=K(y, x)^{*}$ for all $x, y \in X$, that is, $K$ is Hermitian. Then

(2) Let $K(x, x)=0$ and consider (3.5) for arbitrary $y \in X$ and $g, h \in \mathcal{H}$.

$$
[K(x, y) g, h]_{\mathcal{H}}+[K(y, x) h, g]_{\mathcal{H}} \geq-[K(y, y) g, g]_{\mathcal{H}}
$$

We claim that

$$
[K(x, y) g, h]_{\mathcal{H}}+[K(y, x) h, g]_{\mathcal{H}}=0 .
$$

Indeed, taking into account the $K$ is 1-positive, we have two choices only: if $[K(y, y) g, g]_{\mathcal{H}}=0$, from $(3.6)$ it follows that $[K(x, y) g, h]_{\mathcal{H}}+[K(y, x) h, g]_{\mathcal{H}} \geq$ 0 and then, replacing $g$ by $-g$ we obtain the opposite inequality, hence (3.7) holds. The second possible choice is $[K(y, y) g, g]_{\mathcal{H}}>0$ when, observing that the rightmost term in (3.6) does not depend on $h$, we can replace $h$ by $t h$, for $t \in \mathbb{R}$. But then, from (3.6) it follows that the only possibility is that (3.7) should hold, since the opposite leads to a contradiction. Thus, (3.7) is proven.

To finish the proof, in (3.7) we replace $g$ by i $g$ and get $[K(x, y) g, h]_{\mathcal{H}}-$ $[K(y, x) h, g]_{\mathcal{H}}=0$ which, in combination with (3.7) implies $[K(x, y) g, h]_{\mathcal{H}}=$ 0 for all $g, h \in \mathcal{H}$, hence $K(x, y)=0$.

The following result is a surrogate of a Schwarz inequality for kernels and it will have a technical role in this article.

Proposition 3.2. Assume that the kernel $K$ is $2 n$-positive for some natural number $n$. Then, for any $x, y_{1}, y_{2}, \ldots, y_{n} \in X$ and any $g_{1}, g_{2}, \ldots, g_{n} \in \mathcal{H}$, the following inequality holds

$$
\sum_{j, k=1}^{n}\left[K\left(x, y_{j}\right) g_{j}, K\left(x, y_{k}\right) g_{k}\right]_{\mathcal{H}} \leq\|K(x, x)\| \sum_{j, k=1}^{n}\left[K\left(y_{k}, y_{j}\right) g_{j}, g_{k}\right]_{\mathcal{H}} \text {. }
$$


Proof. Since $K$ is $2 n$-positive, it follows that for any $x_{1}, \ldots, x_{2 n} \in X$ and $h_{1}, \ldots, h_{2 n} \in \mathcal{H}$ we have

$$
\sum_{j, k=1}^{2 n}\left[K\left(x_{k}, x_{j}\right) h_{j}, h_{k}\right]_{\mathcal{H}} \geq 0 .
$$

For each $k=1, \ldots, n$ we make the following choice

$$
x_{k}=x, \quad x_{n+k}=y_{k}, \quad h_{k}=-K\left(x, y_{k}\right) g_{k}, \quad h_{n+k}=\|K(x, x)\| g_{k},
$$

in (3.9) and get

$$
\begin{aligned}
0 \leq \sum_{j, k=1}^{n}\left[K(x, x) K\left(x, y_{j}\right) g_{j}, K\left(x, y_{k}\right) g_{k}\right]_{\mathcal{H}} \\
\quad-2\|K(x, x)\| \sum_{j, k=1}^{n}\left[K\left(x, y_{j}\right) g_{j}, K\left(x, y_{k}\right) g_{k}\right]_{\mathcal{H}} \\
\quad+\|K(x, x)\|^{2} \sum_{j, k=1}^{n}\left[K\left(y_{k}, y_{j}\right) g_{j}, g_{k}\right]_{\mathcal{H}}
\end{aligned}
$$

Taking into account that, by Lemma 2.2, when applied for $T=K(x, x) \geq 0$ and $h_{j}=K\left(x, y_{j}\right) g_{j}$, have

$$
\begin{aligned}
\sum_{j, k=1}^{n}[K(x, x) & \left.K\left(x, y_{j}\right) g_{j}, K\left(x, y_{k}\right) g_{k}\right]_{\mathcal{H}} \leq\|K(x, x)\| \\
& \times \sum_{j, k=1}^{n}\left[K\left(x, y_{j}\right) g_{j}, K\left(x, y_{k}\right) g_{k}\right]_{\mathcal{H}},
\end{aligned}
$$

which, when used in (3.10), yields

$\|K(x, x)\| \sum_{j, k=1}^{n}\left[K\left(x, y_{j}\right) g_{j}, K\left(x, y_{k}\right) g_{k}\right]_{\mathcal{H}} \leq\|K(x, x)\|^{2} \sum_{j, k=1}^{n}\left[K\left(y_{k}, y_{j}\right) g_{j}, g_{k}\right]_{\mathcal{H}}$,

which, by Lemma 3.1.(2), implies (3.8).

\subsection{Kolmogorov Decompositions}

Given a $\mathcal{B}^{*}(\mathcal{H})$-valued kernel $K$ on a nonempty set $X$, for some VH-space $\mathcal{H}$ on an admissible space $Z$, a Kolmogorov decomposition of $K$ is, by definition, a pair $(V ; \mathcal{K})$, subject to the following conditions:

$(\mathrm{kd1}) \mathcal{K}$ is a VH-space over the same admissible space $Z$.

$(\mathrm{kd} 2) V: X \rightarrow \mathcal{B}^{*}(\mathcal{H}, \mathcal{K})$ satisfies $K(y, x)=V^{*}(y) V(x)$ for all $x, y \in X$.

If, in addition, the Kolmogorov decomposition satisfies the following condition

$(\mathrm{kd} 3) \operatorname{Lin} V(X) \mathcal{H}$ is dense in $\mathcal{K}$.

then it is called minimal.

Two Kolmogorov decompositions $(V ; \mathcal{K})$ and $\left(V^{\prime} ; \mathcal{K}^{\prime}\right)$ of the same kernel $K$ are called unitary equivalent if there exists a unitary operator $U: \mathcal{K} \rightarrow \mathcal{K}^{\prime}$ such that $U V(x)=V^{\prime}(x)$ for all $x \in X$. 
Theorem 3.3. Given a $\mathcal{B}^{*}(\mathcal{H})$-valued kernel $K$, for some $V H$-space $\mathcal{H}$ on an admissible space $Z$, on a nonempty set $X$, the following assertions are equivalent:

(1) $K$ is positive semidefinite.

(2) $K$ admits a Kolmogorov decomposition $(V ; \mathcal{K})$.

In addition, the Kolmogorov decomposition $(V ; \mathcal{K})$ can always be chosen minimal and, in this case, it is unique up to unitary equivalence.

Proof. $(1) \Rightarrow(2)$. Assuming that $K$ is positive semidefinite, by Lemma 3.1.(1) it follows that $K$ is Hermitian, that is, $K(x, y)^{*}=K(y, x)$ for all $x, y \in X$. With the notation as in Sect. 3.1 we consider the convolution operator $K$ defined at $(3.3)$ and let $\mathcal{F}_{0}=\mathcal{F}_{0}(X ; \mathcal{H})$ be its range, more precisely,

$$
\begin{aligned}
\mathcal{F}_{0} & =\{f \in \mathcal{F} \mid f=K g \text { for some } g \in \mathcal{G}\} \\
& =\left\{f \in \mathcal{F} \mid f(y)=\sum_{x \in X} K(y, x) g(x) \text { for some } g \in \mathcal{G} \text { and all } y \in X\right\}
\end{aligned}
$$

A pairing $[\cdot, \cdot]_{\mathcal{F}_{0}}: X \times X \rightarrow Z$ can be defined by

$$
\begin{aligned}
{[e, f]_{\mathcal{F}_{0}} } & =[g, h]_{K}=[K g, h]_{\mathcal{G}}=\sum_{y \in X}[e(y), h(y)]_{\mathcal{H}} \\
& =\sum_{x, y \in X}[K(y, x) g(x), h(y)]_{\mathcal{H}},
\end{aligned}
$$

where $f=K h$ and $e=K g$ for some $g, h \in \mathcal{G}$, that is, $g$ and $h$ are finitely supported $\mathcal{H}$-valued functions on $X$. We observe that

$$
\begin{aligned}
{[e, f]_{\mathcal{F}_{0}} } & =\sum_{y \in X}[e(y), h(y)]_{\mathcal{H}}=\sum_{x, y \in X}[K(y, x) g(x), h(y)]_{\mathcal{H}} \\
& =\sum_{x, y \in X}[g(x), K(x, y) h(y)]_{\mathcal{H}}=\sum_{x \in X}[g(x), f(x)]_{\mathcal{H}},
\end{aligned}
$$

which shows that the definition in (3.12) is correct (that is, independent of $g$ and $h$ such that $e=K g$ and $f=K h)$.

We claim that $[\cdot, \cdot]_{\mathcal{F}_{0}}$ is a $Z$-valued inner product, that is, it satisfies all the requirements (ve1)-(ve3). The only fact that needs a proof is $[f, f]_{\mathcal{F}_{0}}=0$ implies $f=0$. To see this we use Lemma 2.1 and first get that $\left[f, f^{\prime}\right]_{\mathcal{F}_{0}}=0$ for all $f^{\prime} \in \mathcal{F}_{0}$. For each $x \in X$ and each $h \in \mathcal{H}$ let $\delta_{x} h \in \mathcal{G}$ denote the function

$$
\left(\delta_{x} h\right)(y)= \begin{cases}h, & \text { if } y=x \\ 0, & \text { if } y \neq x\end{cases}
$$

(A correct notation would be $\delta_{x} h=\delta_{x} \otimes h$, when identifying $\mathcal{G}(X ; \mathcal{H})$ with $\mathcal{G}(X ; \mathbb{C}) \otimes \mathcal{H}$, but we resisted the temptation of using it in order to keep the notation simpler.) Letting $f^{\prime}=K \delta_{x} h$ we have

$$
0=\left[f, f^{\prime}\right]_{\mathcal{F}_{0}}=\left[f, K \delta_{x} h\right]_{\mathcal{G}}=\sum_{y \in X}\left[f(y),\left(\delta_{x} h\right)(y)\right]_{\mathcal{H}}=[f(x), h]_{\mathcal{H}},
$$

hence, since $h \in \mathcal{H}$ and $x \in X$ are arbitrary, it follows that $f=0$. 
Thus, $\left(\mathcal{F}_{0} ;[\cdot, \cdot]_{\mathcal{F}_{0}}\right)$ is a VE-space that can be completed to a VH-space that we denote by $\mathcal{K}$, that contains $\mathcal{F}_{0}$ as a dense linear manifold. For each $x \in X$ we define $V(x): \mathcal{H} \rightarrow \mathcal{F}_{0}$ by

$$
V(x) h=K \delta_{x} h, \quad h \in \mathcal{H} .
$$

Actually, there is an even more explicit way of expressing $V(x)$, namely,

$$
\begin{aligned}
(V(x) h)(y)=\left(K \delta_{x} h\right)(y) & =\sum_{z \in X} K(y, z)\left(\delta_{x} h\right)(z) \\
& =K(y, x) h, \quad y \in X .
\end{aligned}
$$

We first show that $V(x)$ is a bounded operator from the VH-space $\mathcal{H}$ to the VE-space $\mathcal{F}_{0}$. Indeed,

$$
\begin{aligned}
{[V(x) h, V(x) h]_{\mathcal{F}_{0}} } & =\left[K \delta_{x} h, K \delta_{x} h\right]_{\mathcal{F}_{0}}=\sum_{y, z \in X}\left[K(y, z)\left(\delta_{x} h\right)(z),\left(\delta_{x} h\right)(y)\right]_{\mathcal{H}} \\
& =[K(x, x) h, h]_{\mathcal{H}} \leq\|K(x, x)\|[h, h]_{\mathcal{H}} .
\end{aligned}
$$

Thus, $V(x)$ is bounded and hence can be uniquely extended by continuity to an operator $V(x) \in \mathcal{B}(\mathcal{H}, \mathcal{K})$.

We now show that $V(x)$ is adjointable for all $x \in X$. To see this, let us fix $x \in X$ and take $h \in \mathcal{H}$ and $f \in \mathcal{F}_{0}$ arbitrary. Then,

$$
[V(x) h, f]_{\mathcal{F}_{0}}=\sum_{y \in X}\left[\left(\delta_{x} h\right)(y), f(y)\right]_{\mathcal{H}}=[h, f(x)]_{\mathcal{H}},
$$

which shows that, if $V(x)$ is adjointable then its adjoint, when restricted to $\mathcal{F}_{0}$ should be $\mathcal{F}_{0} \ni f \mapsto f(x)=W(x) f \in \mathcal{H}$. We prove that $W(x)$ is bounded as a linear operator from the VE-space $\mathcal{F}_{0}$ to the VH-space $\mathcal{H}$. To this end, let $f \in \mathcal{F}_{0}$ be arbitrary, hence $f=K g$ for some finitely supported $g$. Then

$$
\begin{aligned}
{[W(x) f, W(x)]_{\mathcal{H}} } & =[f(x), f(x)]_{\mathcal{H}}=\left[\sum_{z \in X} K(x, z) g(z), \sum_{y \in X} K(x, y) g(y)\right]_{\mathcal{H}} \\
& =\sum_{z, y \in X}[K(x, z) g(z), K(x, y) g(y)]_{\mathcal{H}}
\end{aligned}
$$

and, by Proposition 3.2, we get

$$
\leq\|K(x, x)\| \sum_{z, y \in X}[K(y, z) g(z), g(y)]_{\mathcal{H}}=\|K(x, x)\|[f, f]_{\mathcal{F}_{0}} .
$$

This proves that $W(x)$ is bounded and hence can be extended uniquely, by continuity, to an operator $W(x) \in \mathcal{B}(\mathcal{K}, \mathcal{H})$. By (3.16) it follows that $V(x)$ is adjointable and $W(x)=V(x)^{*}$ for all $x \in X$, more precisely,

$$
V(x)^{*} f=f(x), \quad f \in \mathcal{F}_{0} .
$$

On the other hand, for any $x, y \in X$, by (3.17) and (3.15), we have

$$
V(y)^{*} V(x) h=(V(x) h)(y)=K(y, x) h, \quad h \in \mathcal{H},
$$

hence $(V ; \mathcal{K})$ is a Kolmogorov decomposition of $K$. We prove that it is minimal as well. To see this, note that for any $g \in \mathcal{G}$, with the notation as in 
(3.13), we have

$$
g=\sum_{x \in \operatorname{supp}(g)} \delta_{x} g(x),
$$

hence, by (3.14), the linear span of $V(X) \mathcal{H}$ equals $\mathcal{F}_{0}$ which is dense in $\mathcal{K}$.

The uniqueness of the minimal Kolmogorov decomposition $(V ; \mathcal{K})$ just constructed follows in the usual way: if $\left(V^{\prime} ; \mathcal{K}^{\prime}\right)$ is another minimal Kolmogorov decomposition of $K$, for arbitrary $x_{1}, \ldots, x_{m}, y_{1}, \ldots, y_{n} \in X$ and arbitrary $g_{1}, \ldots, g_{m}, h_{1}, \ldots, h_{n} \in \mathcal{H}$, we have

$$
\begin{aligned}
{\left[\sum_{j=1}^{m} V\left(x_{j}\right) g_{j}, \sum_{k=1}^{m} V\left(y_{k}\right) h_{k}\right]_{\mathcal{K}} } & =\sum_{j=1}^{m} \sum_{k=1}^{n}\left[V\left(y_{k}\right)^{*} V\left(x_{j}\right) g_{j}, h_{k}\right]_{\mathcal{H}} \\
& =\sum_{k=1}^{m} \sum_{j=1}^{n}\left[K\left(y_{k}, x_{j}\right) g_{j}, h_{k}\right]_{\mathcal{H}} \\
& =\sum_{j=1}^{m} \sum_{k=1}^{n}\left[V^{\prime}\left(y_{k}\right)^{*} V^{\prime}\left(x_{j}\right) g_{j}, h_{k}\right]_{\mathcal{H}} \\
& =\left[\sum_{j=1}^{m} V^{\prime}\left(x_{j}\right) g_{j}, \sum_{k=1}^{m} V^{\prime}\left(y_{k}\right) h_{k}\right]_{\mathcal{K}^{\prime}}
\end{aligned}
$$

hence $U: \operatorname{Lin} V(X) \mathcal{H} \rightarrow \operatorname{Lin} V^{\prime}(X) \mathcal{H}$ defined by

$$
\sum_{j=1}^{m} V\left(x_{j}\right) g_{j} \mapsto \sum_{j=1}^{m} V^{\prime}\left(x_{j}\right) g_{j}
$$

is a linear operator, correctly defined, isometric, densely defined, and with dense range. Thus, $U$ extends uniquely to a unitary operator $U \in \mathcal{B}^{*}\left(\mathcal{K}, \mathcal{K}^{\prime}\right)$ and $U V(x)=V^{\prime}(x)$ for all $x \in X$, by construction.

$(2) \Rightarrow(1)$. This is proven exactly as in the classical case:

$$
\begin{aligned}
\sum_{j, k=1}^{n}\left[K\left(x_{k}, x_{j}\right) h_{j}, h_{k}\right]_{\mathcal{H}} & =\sum_{j, k=1}^{n}\left[V\left(x_{k}\right)^{*} V\left(x_{j}\right) h_{j}, h_{k}\right]_{\mathcal{H}} \\
& =\left[\sum_{j=1}^{n} V\left(x_{j}\right) h_{j}, \sum_{j=1}^{n} V\left(x_{j}\right) h_{j}\right]_{\mathcal{H}} \geq 0
\end{aligned}
$$

for all $n \in \mathbb{N}, x_{1}, \ldots, x_{n} \in X$, and $h_{1}, \ldots, h_{n} \in \mathcal{H}$.

\subsection{Reproducing Kernel VH-Spaces}

Let $\mathcal{H}$ be a VH-space over the admissible space $Z$, and let $X$ be a nonempty set. As in Sect. 3.1, we consider the complex vector space $\mathcal{F}(X ; \mathcal{H})$ of all functions $f: X \rightarrow \mathcal{H}$. A VH-space $\mathcal{R}$, over the same admissible space $Z$, is called an $\mathcal{H}$-reproducing kernel $V H$-space on $X$ if there exists a Hermitian kernel $K: X \times X \rightarrow \mathcal{B}^{*}(\mathcal{H})$ such that the following axioms are satisfied:

(rk1) $\mathcal{R}$ is a subspace of $\mathcal{F}(X ; \mathcal{H})$, with all algebraic operations. 
(rk2) For all $x \in X$ and all $h \in \mathcal{H}$, the $\mathcal{H}$-valued function $K_{x} h=K(\cdot, x) h \in$ $\mathcal{R}$.

(rk3) For all $f \in \mathcal{R}$ we have $[f(x), h]_{\mathcal{H}}=\left[f, K_{x} h\right]_{\mathcal{R}}$, for all $x \in X$ and $h \in \mathcal{H}$. In addition, as a consequence of ( $\mathrm{rk} 3)$, the following minimality property holds as well:

(rk4) $\operatorname{Lin}\left\{K_{x} h \mid x \in X, h \in \mathcal{H}\right\}$ is dense in $\mathcal{R}$.

Proposition 3.4. Assume that $\mathcal{R}$ is an $\mathcal{H}$-reproducing kernel VH-space on $X$ with kernel $K$.

(a) $K$ is positive semidefinite and uniquely determined by $\mathcal{R}$.

(b) $\mathcal{R}$ is uniquely determined by $K$.

Proof. (a) Using the reproducing axiom (rk3) it follows

$$
\begin{aligned}
\sum_{j, k=1}^{n}\left[K\left(x_{k}, x_{j}\right) h_{j}, h_{k}\right]_{\mathcal{H}} & =\sum_{j, k=1}^{n}\left[K_{x_{j}} h_{j}, K_{x_{k}} h_{k}\right]_{\mathcal{H}} \\
& =\left[\sum_{j=1}^{n} K_{x_{j}} h_{j}, \sum_{j=1}^{n} K_{x_{j}} h_{j}\right]_{\mathcal{H}} \geq 0
\end{aligned}
$$

hence $K$ is positive semidefinite.

On the other hand, by (rk3) it follows that all the functions $K_{x} h, x \in$ $X, h \in \mathcal{H}$ are uniquely determined by $\left(\mathcal{R} ;[\cdot, \cdot]_{\mathcal{R}}\right)$, hence all the operators $K(y, x)=K_{x}(y), x, y \in X$, are uniquely determined.

(b) Let $\mathcal{R}^{\prime}$ be another $\mathcal{H}$-reproducing kernel VH-space on $X$ with kernel $K$. By axiom $(\operatorname{rk} 2)$ and $(\operatorname{rk} 4), \mathcal{R}_{0}=\operatorname{Lin}\left\{K_{x} h \mid x \in X, h \in \mathcal{H}\right\}$ is a linear space that lies and is dense in both of $\mathcal{R}$ and $\mathcal{R}^{\prime}$. By axiom (rk3), the $Z$-valued inner products $[\cdot, \cdot]_{\mathcal{R}}$ and $[\cdot, \cdot]_{\mathcal{R}^{\prime}}$ coincide on $\mathcal{R}_{0}$ and then it is easy to see that, due to the special way in which the topologies on $\mathcal{R}$ and $\mathcal{R}^{\prime}$ are defined (see (2.2) and (2.3)) and the density of $\mathcal{R}_{0}$, we actually have $\mathcal{R}=\mathcal{R}^{\prime}$ as VH-spaces.

Consequently, given $\mathcal{R}$ an $\mathcal{H}$-reproducing kernel VH-space on $X$ we can talk about the $\mathcal{H}$-reproducing kernel $K$ corresponding to $\mathcal{R}$.

The following theorem adds one more equivalent characterization of VH-spaces operator valued positive semidefinite kernels in terms of reproducing kernel VH-spaces. Our point of view is to obtain this equivalent statement through Kolmogorov decompositions.

Theorem 3.5. Let $\mathcal{H}$ be a VH-space over the admissible space $Z, X$ a nonempty set, and $K: X \times X \rightarrow \mathcal{B}^{*}(\mathcal{H})$ a Hermitian kernel. The following assertions are equivalent:

(1) $K$ is positive semidefinite.

(2) K has a Kolmogorov decomposition.

(3) $K$ is the $\mathcal{H}$-reproducing kernel on $X$ of a VH-space $\mathcal{R}$.

Proof. The equivalence $(1) \Leftrightarrow(2)$ was proven in Theorem 3.3. Even though we already have the implication $(3) \Rightarrow(1)$ by Proposition 3.4 , we prefer to prove the equivalence of assertions (2) and (3) independently of this, in order to 
show explicitly both ways of the connection between Kolmogorov decompositions and reproducing kernel VH-spaces.

$(2) \Rightarrow(3)$. Let $(\mathcal{K} ; V)$ be a Kolmogorov decomposition of $K$. As shown by Theorem 3.3, without loss of generality we can assume it to be minimal as well. Define

$$
\mathcal{R}=\left\{V(\cdot)^{*} f \mid f \in \mathcal{K}\right\},
$$

that is, $\mathcal{R}$ consists of all functions $X \ni x \mapsto V(x)^{*} f \in \mathcal{H}$, in particular $\mathcal{R} \subseteq \mathcal{F}(X ; \mathcal{H})$, and we endow $\mathcal{R}$ with the algebraic operations inherited from the complex vector space $\mathcal{F}(X ; \mathcal{H})$.

We want to show that the correspondence

$$
\mathcal{K} \ni f \mapsto U f=V(\cdot)^{*} f \in \mathcal{R}
$$

is bijective. By the definition of $\mathcal{R}$, this correspondence is surjective. In order to verify that it is injective as well, let $f, g \in \mathcal{K}$ be such that $V^{*}(\cdot) f=V^{*}(\cdot) g$. Then, for all $x \in X$ and all $h \in \mathcal{H}$ we have

$$
\left[V(x)^{*} f, h\right]_{\mathcal{H}}=\left[V(x)^{*} g, h\right]_{\mathcal{H}},
$$

equivalently,

$$
[f-g, V(x) h]_{\mathcal{K}}=0, \quad x \in X, h \in \mathcal{H} .
$$

By the minimality of the Kolmogorov decomposition $(\mathcal{K} ; V)$ it follows that $g=f$. Thus, $U$ is a bijection.

Clearly, the bijective map $U$ defined at (3.20) is linear, hence a linear isomorphism of complex vector spaces $\mathcal{K} \rightarrow \mathcal{R}$. On $\mathcal{R}$ we introduce a $Z$-valued pairing

$$
[U f, U g]_{\mathcal{R}}=\left[V(\cdot)^{*} f, V(\cdot)^{*} g\right]_{\mathcal{R}}=[f, g]_{\mathcal{K}}, \quad f, g \in \mathcal{K} .
$$

Since $\left(\mathcal{K} ;[\cdot, \cdot]_{\mathcal{K}}\right)$ is a VH-space over $Z$, it follows that $\left(\mathcal{R} ;[\cdot, \cdot]_{\mathcal{R}}\right)$ is a VH-space over $Z$ : just note that, by (3.21) we transported the $Z$-gramian from $\mathcal{K}$ to $\mathcal{R}$ or, in other words, we have defined on $\mathcal{R}$ the $Z$-gramian that makes the linear isomorphism $U$ a unitary operator between the VH-spaces $\mathcal{K}$ and $\mathcal{R}$.

We show that $\left(\mathcal{R} ;[\cdot, \cdot]_{\mathcal{R}}\right)$ is an $\mathcal{H}$-reproducing kernel VH-space with corresponding reproducing kernel $K$. By definition, $\mathcal{R} \subseteq \mathcal{F}(X ; \mathcal{H})$. On the other hand, since

$$
K_{x}(y) h=K(y, x) h=V(y)^{*} V(x) h, \text { for all } x, y \in X \text { and all } h \in \mathcal{H},
$$

taking into account that $V(x) h \in \mathcal{K}$, by (3.19) it follows that $K_{x} \in \mathcal{R}$ for all $x \in X$. Further, for all $f \in \mathcal{R}, x \in X$, and $h \in \mathcal{H}$, we have

$$
\begin{aligned}
{\left[f, K_{x} h\right]_{\mathcal{R}} } & =\left[V(\cdot)^{*} g, K_{x} h\right]_{\mathcal{R}}=\left[V(\cdot)^{*} g, V(\cdot)^{*} V(x) h\right]_{\mathcal{R}} \\
& =[g, V(x) h]_{\mathcal{K}}=\left[V(x)^{*} g, h\right]_{\mathcal{H}}=[f, h]_{\mathcal{H}},
\end{aligned}
$$

where $g \in \mathcal{K}$ is the unique vector such that $V(x)^{*} g=f$, which shows that $\mathcal{R}$ satisfies the reproducing axiom as well. Finally, taking into account the minimality of the Kolmogorov decomposition $(\mathcal{K} ; V)$ and the definition (3.19), it follows that $\overline{\operatorname{Lin}\left\{K_{x} \mid x \in X\right\}}=\mathcal{R}$. Thus, we finish proving that $\left(\mathcal{R} ;[\cdot, \cdot]_{\mathcal{R}}\right)$ is an $\mathcal{H}$-reproducing kernel VH-space with reproducing kernel $K$. 
$(3) \Rightarrow(2)$. Assume that $\left(\mathcal{R} ;[\cdot, \cdot]_{\mathcal{R}}\right)$ is an $\mathcal{H}$-reproducing kernel VH-space on $X$, with reproducing kernel $K$. We let $\mathcal{K}=\mathcal{R}$ and define

$$
V(x) h=K_{x} h, \quad x \in X, h \in \mathcal{H} .
$$

Note that $V(x): \mathcal{H} \rightarrow \mathcal{K}$ is linear for all $x \in X$.

We have to show that $V(x) \in \mathcal{B}^{*}(\mathcal{H}, \mathcal{K})$ for all $x \in X$. To see this, first note that, by the reproducing property, for all $h \in \mathcal{H}$ we have

$$
[V(x) h, V(x) h]_{\mathcal{K}}=\left[K_{x} h, K_{x} h\right]_{\mathcal{R}}=[K(x, x) h, h]_{\mathcal{H}} \leq\|K(x, x)\|[h, h]_{\mathcal{H}},
$$

hence $V(x)$ is bounded for all $x \in X$. On the other hand,

$$
[f, V(x) h]_{\mathcal{K}}=\left[f, K_{x} h\right]_{\mathcal{R}}=[f(x), h]_{\mathcal{H}}, \quad x \in X, h \in \mathcal{H} .
$$

Let us then, for fixed $x \in X$, consider the linear operator $W(x): \mathcal{R}=\mathcal{K} \rightarrow \mathcal{H}$ defined by $W(x) f=f(x)$ for all $f \in \mathcal{R}=\mathcal{K}$. In order to show that $W(x)$ is bounded, by the minimality property (rk4) it follows that it is sufficient to consider only functions $f \in \operatorname{Lin}\left\{K_{x} h \mid x \in X, h \in \mathcal{H}\right\}$. Thus, if $f=$ $K_{x_{1}} h_{1}+\cdots+K_{x_{n}} h_{n}$ it follows that

$$
\begin{aligned}
{[W(x) f, W(x) f]_{\mathcal{H}} } & =[f(x), f(x)]_{\mathcal{H}}=\left[\sum_{j=1}^{n} K\left(x, x_{j}\right) h_{j}, \sum_{k=1}^{n} K\left(x, x_{k}\right) h_{k}\right]_{\mathcal{H}} \\
& \leq\|K(x, x)\| \sum_{j, k=1}^{n}\left[K\left(x_{k}, x_{j}\right) h_{j}, h_{k}\right]_{\mathcal{H}},
\end{aligned}
$$

where the inequality follows by Proposition 3.2. Since, by the reproducing axiom, we have

$$
\begin{aligned}
{[f, f]_{\mathcal{R}} } & =\sum_{j, k=1}^{n}\left[K_{x_{j}} h_{j}, K_{x_{k}} h_{k}\right]_{\mathcal{R}} \\
& =\sum_{j, k=1}^{n}\left[K_{x_{j}}\left(x_{k}\right) h_{j}, h_{k}\right]_{\mathcal{H}}=\sum_{j, k=1}^{n}\left[K\left(x_{k}, x_{j}\right) h_{j}, h_{k}\right]_{\mathcal{H}}
\end{aligned}
$$

it follows that, for all $f \in \operatorname{Lin}\left\{K_{x} h \mid x \in X, h \in \mathcal{H}\right\}$, we have

$$
[W(x) f, W(x) f]_{\mathcal{H}} \leq\|K(x, x)\|[f, f]_{\mathcal{R}} .
$$

Thus, $W(x)$ is bounded on a dense linear manifold of $\mathcal{R}$ and hence it extends by continuity to an operator $W(x) \in \mathcal{B}(\mathcal{H}, \mathcal{K})$. From (3.23) we conclude that $V(x)$ is adjointable and $V(x)^{*}=W(x)$ for all $x \in X$.

Finally, by the reproducing axiom, for all $x, y \in X$ and all $h, g \in \mathcal{H}$ we have

$$
\left[V(y)^{*} V(x) h, g\right]_{\mathcal{H}}=[V(x) h, V(y) g]_{\mathcal{R}}=\left[K_{x} h, K_{y} g\right]_{\mathcal{R}}=[K(y, x) h, g]_{\mathcal{H}},
$$

hence $V(y)^{*} V(x)=K(y, x)$ for all $x, y \in X$. Thus, $(\mathcal{K} ; V)$ is a Kolmogorov decomposition of $K$ (actually, a minimal one).

Given $K: X \times X \rightarrow \mathcal{B}^{*}(\mathcal{H})$ a positive semidefinite kernel, as a consequence of Theorem 3.5 and statement (b) in Proposition 3.4, we can denote, without any ambiguity, by $\mathcal{R}_{K}$ the unique $\mathcal{H}$-reproducing kernel $\mathrm{VH}$-space on $X$ associated to $K$. 
Remark 3.6. There is another by-product of the proofs of Theorem 3.3 and that of Theorem 3.5 namely, that, up to the abstract completion, the construction in Theorem 3.3 is essentially an $\mathcal{H}$-reproducing kernel one. More precisely, with the notation as in the proof of the implication $(1) \Rightarrow(2)$ of Theorem 3.3, we first note that, for arbitrary $f \in \mathcal{F}(X ; \mathcal{H}), f=K g$ with $g \in \mathcal{G}(X ; \mathcal{H})$, we have

$$
f=\sum_{x \in X} K(y, x) g(x)=\sum_{x \in X} K_{x}(y) g(x),
$$

hence $\mathcal{F}_{0}(X ; \mathcal{H})=\operatorname{Lin}\left\{K_{x} h \mid x \in X, h \in \mathcal{H}\right\}$. Then, for arbitrary $f \in \mathcal{F}_{0}$ we have

$$
\begin{aligned}
{\left[f, K_{x} h\right]_{\mathcal{K}} } & =\left[f, K_{x} h\right]_{\mathcal{F}_{0}}=\left[f, K \delta_{x} h\right]_{\mathcal{F}_{0}}=\sum_{y \in X}\left[f(y),\left(\delta_{x} h\right)(y)\right]_{\mathcal{H}} \\
& =[f(x), h]_{\mathcal{H}}=\left[f, K_{x} h\right]_{\mathcal{R}(K)}, \quad x \in X, h \in \mathcal{H},
\end{aligned}
$$

hence $[\cdot, \cdot]_{\mathcal{K}}=[\cdot, \cdot]_{\mathcal{R}(K)}$ on $\mathcal{F}_{0}(X ; \mathcal{H})=\operatorname{Lin}\left\{K_{x} h \mid x \in X, h \in \mathcal{H}\right\}$, that is dense in both $\mathcal{K}$ and $\mathcal{R}(K)$. Therefore, we can take $\mathcal{K}=\mathcal{R}(K)$ as the completion of $\mathcal{F}_{0}(X ; \mathcal{H})$ to a VH-space, with the advantage that it consists entirely of $\mathcal{H}$-valued functions on $X$ and hence, it is very "concrete".

This fact can be put in the following way as well: the completion of the $V E$-space $\mathcal{F}_{0}(X ; \mathcal{H})$ can be performed within $\mathcal{F}(X ; \mathcal{H})$, and this is exactly the $\mathcal{H}$-reproducing kernel $V H$-space $\mathcal{H}(K)$. In order to prove this statement there are, at least, two different paths. One way is that we just mentioned, going through the Kolmogorov decomposition $(\mathcal{K} ; V)$ obtained in the proof of Theorem 3.3. Alternatively, there is a more direct way that we can briefly outline: if $\left(f_{j}\right)$ is a net in $\mathcal{F}_{0}(X ; \mathcal{H})$ that is Cauchy with respect to the locally convex topology of the VE-space $\mathcal{F}_{0}(X ; \mathcal{H})$, one can prove that for all $x \in X$ the net $\left(f_{j}(x)\right)$ is Cauchy within the VH-space $\mathcal{H}$, which is complete, hence let $f(x) \in \mathcal{H}$ be its limit. In this way, we obtained $f \in \mathcal{F}(X ; \mathcal{H})$ and it remains to prove that $f \in \mathcal{H}(K)$ and the net $\left(f_{j}\right)$ converges to $f$ in $\mathcal{H}(K)$. We leave the details to the reader.

\section{Kernels Invariant under Actions of $*$-Semigroups}

Let $X$ be a nonempty set, a (multiplicative) semigroup $\Gamma$, and an action of $\Gamma$ on $X$, denoted by $\xi \cdot x$, for all $\xi \in \Gamma$ and all $x \in X$. By definition, we have

$$
\alpha \cdot(\beta \cdot x)=(\alpha \beta) \cdot x \text { for all } \alpha, \beta \in \Gamma \text { and all } x \in X .
$$

Alternatively, this means that we have a semigroup morphism $\Gamma \ni \xi \mapsto \xi \cdot \epsilon$ $G(X)$, where $G(X)$ denotes the semigroup, with respect to composition, of all maps $X \rightarrow X$. In case the semigroups $\Gamma$ has a unit $\epsilon$, the action is called unital if $\epsilon \cdot x=x$ for all $x \in X$, equivalently, $\epsilon \cdot=\operatorname{Id}_{X}$.

We assume further that $\Gamma$ is a $*$-semigroup, that is, there is an involution $*$ on $\Gamma$; this means that $(\xi \eta)^{*}=\eta^{*} \xi^{*}$ and $\left(\xi^{*}\right)^{*}=\xi$ for all $\xi, \eta \in \Gamma$. Note that, in case $\Gamma$ has a unit $\epsilon$ then $\epsilon^{*}=\epsilon$. 
Given a VH-space $\mathcal{H}$ we are interested in those Hermitian kernels $K: X \times X \rightarrow \mathcal{B}^{*}(\mathcal{H})$ that are invariant under the action of $\Gamma$ on $X$, that is,

$$
K(y, \xi \cdot x)=K\left(\xi^{*} \cdot y, x\right) \text { for all } x, y \in X \text { and all } \xi \in \Gamma \text {. }
$$

A triple $(\mathcal{K} ; \pi ; V)$ is called an invariant Kolmogorov decomposition, of the kernel $K$ and the action of $\Gamma$ on $X$, if:

(ikd1) $(\mathcal{K} ; V)$ is a Kolmogorov decomposition of the kernel $K$.

$(\operatorname{ikd} 2) \pi: \Gamma \rightarrow \mathcal{B}^{*}(\mathcal{K})$ is a $*$-representation, that is, a multiplicative *-morphism.

(ikd3) $V$ and $\pi$ are related by the formula: $V(\xi \cdot x)=\pi(\xi) V(x)$, for all $x \in X, \xi \in \Gamma$.

In order to explain this definition, let $(\mathcal{K} ; \pi ; V)$ be an invariant Kolmogorov decomposition of the kernel $K$. Since $(\mathcal{K} ; V)$ is a Kolmogorov decomposition and taking into account the axiom (ikd3), for all $x, y \in X$ and all $\xi \in \Gamma$, we have

$$
\begin{aligned}
K(y, \xi \cdot x) & =V(y)^{*} V(\xi \cdot x)=V(y)^{*} \pi(\xi) V(x) \\
& =\left(\pi\left(\xi^{*}\right) V(y)\right)^{*} V(x)=K\left(\xi^{*} \cdot y, x\right),
\end{aligned}
$$

hence $K$ is invariant under the action of $\Gamma$ on $X$.

If, in addition to the axioms (idk1)-(idk3), the triple $(\mathcal{K} ; \pi ; V)$ has also the property

$(\mathrm{ikd} 4) \operatorname{Lin} V(X) \mathcal{H}$ is dense in $\mathcal{K}$,

that is, the Kolmogorov decomposition $(\mathcal{K} ; V)$ is minimal, then $(\mathcal{K} ; \pi ; V)$ is called a minimal invariant Kolmogorov decomposition of $K$ and the action of $\Gamma$ on $X$.

The next proposition shows that minimal invariant Kolmogorov decompositions have a built-in linearity property.

Proposition 4.1. Assume that, given an VH-operator valued kernel $K$, invariant under the action of the $*$-semigroup $\Gamma$ on $X$, for some fixed $\alpha, \beta, \gamma \in \Gamma$ we have $K(y, \alpha \cdot x)+K(y, \beta \cdot x)=K(y, \gamma \cdot x)$ for all $x, y \in X$. Then for any minimal invariant Kolmogorov decomposition $(\mathcal{K} ; \pi ; V)$ of $K$, the representation satisfies $\pi(\alpha)+\pi(\beta)=\pi(\gamma)$.

Proof. For any $x, y \in X$ and any $h, k \in \mathcal{H}$ we have

$$
\begin{aligned}
{[(\pi(\alpha)+\pi(\beta)) V(x) h, V(y) k]_{\mathcal{H}} } & =\left[V^{*}(y) \pi(\alpha) V(x) h+V^{*}(y) \pi(\beta) V(x) h, k\right]_{\mathcal{H}} \\
& =[K(y, \alpha \cdot x) h+K(y, \beta \cdot y), k]_{\mathcal{H}} \\
& =[K(y \cdot \gamma \cdot x) h, k]_{\mathcal{H}} \\
& =\left[V(y)^{*} \pi(\gamma) V(x) h, k\right]_{\mathcal{H}} \\
& =[\pi(\gamma) V(x) h, V(y) k]_{\mathcal{H}},
\end{aligned}
$$

hence, since $V(X) \mathcal{H}$ is total in $\mathcal{H}$, it follows that $\pi(\alpha)+\pi(\beta)=\pi(\gamma)$.

Two invariant Kolmogorov decompositions $(\mathcal{K} ; \pi ; V)$ and $\left(\mathcal{K}^{\prime} ; \pi^{\prime} ; V^{\prime}\right)$, of the same Hermitian kernel $K$, are called unitary equivalent if there exists a unitary operator $U: \mathcal{K} \rightarrow \mathcal{K}^{\prime}$ such that $U \pi(\xi)=\pi^{\prime}(\xi) U$ for all $\xi \in \Gamma$, and 
$U V(x)=V^{\prime}(x)$ for all $x \in X$. Let us note that, in case both of these invariant Kolmogorov decompositions are minimal, then this is equivalent with the requirement that the Kolmogorov decompositions $(\mathcal{K} ; V)$ and $\left(\mathcal{K}^{\prime} ; V^{\prime}\right)$ are unitary equivalent.

Theorem 4.2. Let $\Gamma$ be a unital $*$-semigroup that acts on the nonempty set $X$, and let $K: X \times X \rightarrow \mathcal{B}^{*}(\mathcal{H})$ be a kernel, for some VH-space $\mathcal{H}$ over an admissible space $Z$. The following assertions are equivalent:

(1) $K$ satisfies the following conditions:

(a) $K$ is positive semidefinite.

(b) $K$ is invariant under the action of $\Gamma$ on $X$, that is, (4.2) holds.

(c) For any $\alpha \in \Gamma$ there exists $c(\alpha) \geq 0$ such that

$$
\sum_{j, k=1}^{n}\left[K\left(\alpha \cdot x_{k}, \alpha \cdot x_{j}\right) h_{j}, h_{k}\right]_{\mathcal{H}} \leq c(\alpha)^{2} \sum_{j, k=1}^{n}\left[K\left(x_{k}, x_{j}\right) h_{j}, h_{k}\right]_{\mathcal{H}},
$$

for $n \in \mathbb{N}$, all $x_{1}, \ldots, x_{n} \in X$, and all $h_{1}, \ldots, h_{n} \in \mathcal{H}$.

(2) $K$ has an invariant Kolmogorov decomposition $(\mathcal{K} ; \pi ; V)$.

(3) $K$ admits an $\mathcal{H}$-reproducing kernel $V H$-space $\mathcal{R}$ and there exists $a *$-representation $\rho: \Gamma \rightarrow \mathcal{B}^{*}(\mathcal{R})$ such that $\rho(\xi) K_{x} h=K_{\xi \cdot x} h$ for all $\xi \in \Gamma, x \in X, h \in \mathcal{H}$.

Moreover, in case any of the assertions (1), (2), or (3) holds, then a minimal invariant Kolmogorov decomposition can be constructed, any minimal invariant Kolmogorov decomposition is unique up to unitary equivalence, and the pair $(\mathcal{R} ; \rho)$ as in assertion $(3)$ is uniquely determined by $K$ as well.

Proof. (1) $\Rightarrow(2)$. We consider the notation and the minimal Kolmogorov decomposition $(\mathcal{K} ; V)$ constructed as in the proof of the implication $(1) \Rightarrow(2)$ of Theorem 3.3. For each $\xi \in \Gamma$ we let $\pi(\xi): \mathcal{F} \rightarrow \mathcal{F}$ be defined by

$$
(\pi(\xi) f)(y)=f\left(\xi^{*} \cdot y\right), \quad y \in X, \xi \in \Gamma .
$$

We claim that $\pi(\xi)$ leaves $\mathcal{F}_{0}$ invariant. To see this, let $f \in \mathcal{F}_{0}$, that is, $f=K g$ for some $g \in \mathcal{G}$ or, even more explicitly, by (3.11),

$$
f(y)=\sum_{x \in X} K(y, x) g(x), \quad y \in X .
$$

Then,

$$
\begin{aligned}
f\left(\xi^{*} \cdot y\right) & =\sum_{x \in X} K\left(\xi^{*} \cdot y, x\right) g(x) \\
& =\sum_{x \in X} K(y, \xi \cdot x) g(x)=\sum_{z \in X} K(y, z) g_{\xi}(z),
\end{aligned}
$$

where,

$$
g_{\xi}(z)= \begin{cases}0, & \text { if } \xi \cdot x=z \text { has no solution } x \in X, \\ \sum_{\xi \cdot x=z} g(x), & \text { otherwise. }\end{cases}
$$


Since clearly $g_{\xi} \in \mathcal{G}$, that is, $g_{\xi}$ has finite support, it follows that $\pi(\xi)$ leaves $\mathcal{F}_{0}$ invariant. In the following we denote by the same symbol $\pi(\xi)$ the map $\pi(\xi): \mathcal{F}_{0} \rightarrow \mathcal{F}_{0}$.

In the following we prove that $\pi$ is a representation of the semigroup $\Gamma$ on the complex vector space $\mathcal{F}_{0}$, that is,

$$
\pi(\alpha \beta) f=\pi(\alpha) \pi(\beta) f, \quad \alpha, \beta \in \Gamma, f \in \mathcal{F}_{0} .
$$

To see this, let $f \in \mathcal{F}_{0}$ be fixed and denote $h=\pi(\beta) f$, that is, $h(y)=f\left(\beta^{*} \cdot y\right)$ for all $y \in X$. Then $\pi(\alpha) \pi(\beta) f=\pi(\alpha) h$, that is, $(\pi(\alpha) h)(y)=h\left(\alpha^{*} \cdot y\right)=$ $h\left(\beta^{*} \alpha^{*} \cdot y\right)=h\left((\alpha \beta)^{*} \cdot y\right)=(\pi(\alpha \beta))(y)$, for all $y \in X$, which proves (4.9)

Next we show that $\pi$ is actually a $*$-representation, that is,

$$
\left[\pi(\xi) f, f^{\prime}\right]_{\mathcal{F}_{0}}=\left[f, \pi\left(\xi^{*}\right) f^{\prime}\right]_{\mathcal{F}_{0}}, \quad f, f^{\prime} \in \mathcal{F}_{0} .
$$

To see this, let $f=K g$ and $f^{\prime}=K g^{\prime}$ for some $g, g^{\prime} \in \mathcal{G}$. Then, recalling (3.12) and (4.7),

$$
\begin{aligned}
{\left[\pi(\xi) f, f^{\prime}\right]_{\mathcal{F}_{0}} } & =\sum_{y \in X}\left[f\left(\xi^{*} y\right), g^{\prime}(y)\right]_{\mathcal{H}}=\sum_{x, y \in X}\left[K\left(\xi^{*} \cdot y, x\right) g(x), g^{\prime}(y)\right]_{\mathcal{H}} \\
& =\sum_{x, y \in X}\left[K(y, \xi \cdot x) g(x), g^{\prime}(h)\right]_{\mathcal{H}}=\sum_{x, y \in X}\left[g(x), K(\xi \cdot x, y) g^{\prime}(y)\right]_{\mathcal{H}} \\
& =\sum_{x \in X}\left[g(x), f^{\prime}(\xi \cdot x)\right]_{\mathcal{H}}=\left[f, \pi\left(\xi^{*}\right) f^{\prime}\right]_{\mathcal{H}},
\end{aligned}
$$

and hence the formula (4.10) is proven.

Considering $\mathcal{F}_{0}$ as a VE-space, we prove now that $\pi(\xi)$ is bounded for all $\xi \in \Gamma$. Indeed, let $f=K g$ for some $g \in \mathcal{G}$. Using the definition of $\pi(\xi)$ and the boundedness condition (c), we have

$$
\begin{aligned}
{[\pi(\xi) f, \pi(\xi) f]_{\mathcal{F}_{0}} } & =\left[\pi\left(\xi^{*}\right) \pi(\xi) f, f\right]_{\mathcal{F}_{0}}=\left[\pi\left(\xi^{*} \xi\right) f, f\right]_{\mathcal{F}_{0}} \\
& =\sum_{x, y \in X}\left[K\left(\xi^{*} \xi \cdot y, x\right) g(x), g(y)\right]_{\mathcal{H}} \\
& =\sum_{x, y \in X}[K(\xi \cdot y, \xi \cdot x) g(x), g(y)]_{\mathcal{H}} \\
& \leq c(\xi)^{2} \sum_{x, y \in X}[K(y, x) g(x), g(y)]_{\mathcal{H}} \\
& =c(\xi)^{2}[f, f]_{\mathcal{F}_{0}},
\end{aligned}
$$

and hence the boundedness of $\pi(\xi)$ is proven. This implies that $\pi(\xi)$ can be uniquely extended by continuity to an operator $\pi(\xi) \in \mathcal{B}(\mathcal{H})$. In addition, since $\pi\left(\xi^{*}\right)$ also extends by continuity to an operator $\pi\left(\xi^{*}\right) \in \mathcal{B}(\mathcal{H})$ and taking into account (4.10), it follows that $\pi(\xi)$ is adjointable and $\pi\left(\xi^{*}\right)=\pi(\xi)^{*}$. We conclude that $\pi$ is a $*$-representation of $\Gamma$ in $\mathcal{B}^{*}(\mathcal{H})$, that is, the axiom (ikd2) holds.

In order to show that the axiom (ikd3) holds as well, we use (3.15). Thus, for all $\xi \in \Gamma, x, y \in X, h \in \mathcal{H}$, and taking into account that $K$ is invariant under the action of $\Gamma$ on $X$, we have 


$$
\begin{aligned}
(V(\xi \cdot x) h)(y) & =K(y, \xi \cdot x) h=K\left(\xi^{*} \cdot y, x\right) h \\
& =(V(x) h)\left(\xi^{*} \cdot y\right)=(\pi(\xi) V(x) h)(y),
\end{aligned}
$$

which proves (ikd3). Thus, $(\mathcal{K} ; \pi ; V)$, here constructed, is an invariant Kolmogorov decomposition of the Hermitian kernel $K$. Note that $(\mathcal{K} ; \pi ; V)$ is minimal, that is, the axiom (ikd4) holds, since the Kolmogorov decomposition $(\mathcal{K} ; V)$ is minimal, by the proof of Theorem 3.3.

In order to prove the uniqueness of the minimal invariant Kolmogorov decomposition, let $\left(\mathcal{K}^{\prime} ; \pi^{\prime} ; V^{\prime}\right)$ be another minimal invariant Kolmogorov decomposition of $K$. We consider the unitary operator $U: \mathcal{K} \rightarrow \mathcal{K}^{\prime}$ defined as in (3.18) and we already know that $U V(x)=V^{\prime}(x)$ for all $x \in X$. Since, for any $\xi \in \Gamma, x \in X$, and $h \in \mathcal{H}$, we have

$$
U \pi(\xi) V(x) h=U V(\xi \cdot x) h=V^{\prime}(\xi \cdot x) h=\pi^{\prime}(\xi) V^{\prime}(x) h=\pi^{\prime}(\xi) U V(x) h,
$$

and taking into account the minimality, it follows that $U \pi(\xi)=\pi^{\prime}(\xi) U$ for all $\xi \in \Gamma$.

$(2) \Rightarrow(1)$. Let $(\mathcal{K} ; \pi ; V)$ be an invariant Kolmogorov decomposition of the kernel $K$. We already know from the proof of Theorem 3.3 that $K$ is positive semidefinite and it was shown in (4.3) that $K$ is invariant under the action of $\Gamma$ on $X$. In order to show that the boundedness condition (c) holds as well, let $\alpha \in \Gamma, n \in \mathbb{N}, x_{1}, \ldots, x_{n} \in X$, and $h_{1}, \ldots, h_{n} \in \mathcal{H}$ be arbitrary. Then

$$
\begin{aligned}
\sum_{j, k=1}^{n}\left[K\left(\alpha \cdot x_{k}, \alpha \cdot x_{j}\right) h_{j}, h_{k}\right]_{\mathcal{H}} & =\sum_{j, k=1}^{n}\left[V\left(x_{k}\right)^{*} \pi\left(\alpha^{*}\right) \pi(\alpha) V\left(x_{j}\right) h_{j}, h_{k}\right]_{\mathcal{H}} \\
& =\sum_{j, k=1}^{n}\left[\pi(\alpha) V\left(x_{j}\right) h_{j}, \pi(\alpha) V\left(x_{k}\right) h_{k}\right]_{\mathcal{H}} \\
& \leq\|\pi(\alpha)\|^{2} \sum_{j, k=1}^{n}\left[V\left(x_{j}\right) h_{j}, V\left(x_{k}\right) h_{k}\right]_{\mathcal{H}} \\
& =\|\pi(\alpha)\|^{2} \sum_{j, k=1}^{n}\left[K\left(x_{k}, x_{j}\right) h_{j}, h_{k}\right]_{\mathcal{H}},
\end{aligned}
$$

and hence (c) holds with $c(\alpha)=\|\pi(\alpha)\| \geq 0$.

$(2) \Rightarrow(3)$. Let $(\mathcal{K} ; \pi ; V)$ be an invariant Kolmogorov decomposition of the kernel $K$ and the action of $\Gamma$ on $X$. Without loss of generality, we can assume that it is minimal. We use the notation and the facts established during the proof of the implication $(2) \Rightarrow(3)$ in Theorem 3.5. Then, for all $x, y \in X$ and $h \in \mathcal{H}$ we have

$$
K_{\xi \cdot x}(y) h=K(y, \xi \cdot x) h=V(y)^{*} V(\xi \cdot x)=V(y)^{*} \pi(\xi) V(x) h,
$$

hence, letting $\rho(\xi)=U \pi(\xi) U^{-1}$, where $U: \mathcal{K} \rightarrow \mathcal{R}$ is the unitary operator defined as in (3.20), we obtain a $*$-representation of $\Gamma$ on the VH-space $\mathcal{R}$ such that $K_{\xi \cdot x}=\rho(\xi) K_{x}$ for all $\xi \in \Gamma$ and $x \in X$.

$(3) \Rightarrow(2)$. Let $(\mathcal{R} ; \rho)$, where $\mathcal{R}=\mathcal{R}(K)$ is the $\mathcal{H}$-reproducing kernel VH-space of $K$ and $\rho: \Gamma \rightarrow \mathcal{B}^{*}(\mathcal{R})$ is a $*$-representation such that $K_{\xi \cdot x}=$ $\rho(\xi) K_{x}$ for all $\xi \in \Gamma$ and $x \in X$. As in the proof of the implication (3) $\Rightarrow(2)$ in 
Theorem 3.5, we show that $(\mathcal{R} ; V)$, where $V$ is defined as in $(3.22)$, is a minimal Kolmogorov decomposition of $K$. Letting $\pi=\rho$, it is then easy to see that $(\mathcal{R} ; \pi ; V)$ is an invariant Kolmogorov decomposition of the kernel $K$ and the action of $\Gamma$ on $X$.

\section{Unification of Two Dilation Theorems in VH-Spaces}

In this section we show that Theorem 4.2 contains both the Loynes generalization of the Sz.-Nagy's Dilation Theorem and the VH-space operator valued generalization of Stinespring's Dilation Theorem.

\subsection{Linearization and Dilation in VH-Spaces}

Recall that a $*$-semigroup is a (multiplicative) semigroup $\Gamma$ on which there exists an involution, denoted by $*$, that is, $\Gamma \ni \gamma \mapsto \gamma^{*} \in \Gamma$ having the properties: $(\beta \gamma)^{*}=\gamma^{*} \beta^{*}$ and $\left(\gamma^{*}\right)^{*}=\gamma$, for all $\beta, \gamma \in \Gamma$. If $\Gamma$ has a unit $\epsilon$ then $\epsilon^{*}=\epsilon$. In case $\Gamma$ is a group and we use the multiplicative notation, we can take $\gamma^{*}=\gamma^{-1}$, but other choices are also possible.

Let $\mathcal{H}$ be a VH-space and consider a family $T=\left\{T_{\xi}\right\}_{\xi \in \Gamma}$ of operators in $\mathcal{B}^{*}(\mathcal{H})$ indexed by a $*$-semigroup $\Gamma$. However, taking into account the framework of this article, it is preferable to think $T$ as a function on $\Gamma$ and valued in $\mathcal{B}^{*}(\mathcal{H})$. Given $n$ an arbitrary natural number, we call $T n$-positive if for any $\eta_{1}, \ldots, \eta_{n} \in \Gamma$ and any $h_{1}, \ldots, h_{n} \in \mathcal{H}$, we have

$$
\sum_{i, j=1}^{n}\left[T_{\eta_{i}^{*} \eta_{j}} h_{j}, h_{i}\right] \geq 0 .
$$

It is easy to see that, if $T$ is $n$-positive then it is $k$-positive for all natural numbers $k \leq n$. $T$ is called positive semidefinite if it is $n$-positive for all natural numbers $n$.

To any map $T: \Gamma \rightarrow \mathcal{B}^{*}(\mathcal{H})$ we associate a kernel $K: \Gamma \times \Gamma \rightarrow \mathcal{B}^{*}(\mathcal{H})$ by

$$
K(\xi, \eta)=T_{\eta^{*} \xi}, \quad \xi, \eta \in \Gamma .
$$

Then, the kernel $K$ is $n$-positive, in the sense of (3.4), if and only if $T$ is $n$-positive, in the sense of (5.1). Consequently, $K$ is positive semidefinite if and only if $T$ is positive semidefinite. Having in mind the Kolmogorov decompositions of Hermitian kernels, see Sect. 3.2, we introduce the following definition. A pair $(\mathcal{K} ; U)$ is called a linearization of $T$ if:

(11) $\mathcal{K}$ is a VH-space on $Z$.

(12) $U: \Gamma \rightarrow \mathcal{B}^{*}(\mathcal{K}, \mathcal{H})$.

(13) $T_{\eta^{*} \xi}=U(\xi) U^{*}(\eta)$ for all $\xi, \eta \in \Gamma$.

Proposition 5.1. Given $\mathcal{H}$ a VH-space on the admissible space $Z$, a unital *-semigroup $\Gamma$, and a map $T: \Gamma \rightarrow \mathcal{B}^{*}(\mathcal{H})$, the following assertions are equivalent:

(1) $T$ is positive semidefinite.

(2) $T$ admits a linearization $(\mathcal{K} ; U)$. 
(3) $T$ yields a reproducing kernel $V H$-space $\mathcal{R}$ over $Z$, that is:

(a) $\mathcal{R}$ consists of functions $f: \Gamma \rightarrow \mathcal{H}$ only.

(b) $T_{\xi} . h \in \mathcal{R}$, that is, the map $\Gamma \ni \eta \mapsto T_{\xi \eta} h \in \mathcal{H}$ belongs to $\mathcal{R}$, for all $\xi \in \Gamma$ and all $h \in \mathcal{H}$.

(c) $[f(\xi), h]_{\mathcal{H}}=\left[f, T_{\xi^{*}}, h\right]_{\mathcal{R}}$ for all $\xi \in \Gamma, h \in \mathcal{H}$, and $f \in \mathcal{R}$.

Proof. The equivalence of (1) and (2) is a consequence of the observations from before applying Theorem 3.3 to the kernel $K(\xi, \eta)=T_{\eta^{*} \xi}$, for $\xi, \eta \in \Gamma$, and letting $U(\xi)=V^{*}(\xi)$, for all $\xi \in \Gamma$.

In order to prove the equivalence of (2) and (3), we apply Theorem 3.5 to the kernel $K(\xi, \eta)=T_{\eta^{*} \xi}$, for $\xi, \eta \in \Gamma$, and observing that $K_{\xi} h=T_{\xi^{*}} . h$, where, $T_{\xi} . h$ denotes the map $\Gamma \ni \eta \mapsto T_{\xi \eta} h \in \mathcal{H}$, for all $\xi \in \Gamma$ all $h \in \mathcal{H}$.

The kernel $K(\xi, \eta)=T_{\eta^{*} \xi}$ has an additional property, namely that it is invariant under the action of $\Gamma$ on itself by left multiplication: $\xi \cdot \eta=\xi \eta$. With the definition as in (4.2), this is proven as follows: for all $\xi, \eta, \gamma \in \Gamma$, we have

$$
K(\eta, \xi \cdot \gamma)=T_{(\xi \cdot \gamma)^{*} \eta}=T_{\gamma^{*}\left(\xi^{*} \cdot \eta\right)}=K\left(\xi^{*} \cdot \eta, \gamma\right) .
$$

Thus, we can consider $T$ having in mind the invariant Kolmogorov decomposition and its reproducing kernel counter-part, as in Theorem 4.2, in order to obtain the following:

Theorem 5.2. Let $\Gamma$ be a *-semigroup with unit $\epsilon$ and $T=\left\{T_{\xi}\right\}_{\xi \in \Gamma} \subseteq \mathcal{B}^{*}(\mathcal{H})$, for some $V H$-space $\mathcal{H}$. The following assertions are equivalent:

(1) T satisfies the following conditions:

(b) $T$ is positive semidefinite as a function on $\Gamma$, in the sense that for any finitely supported family $\left\{g_{\xi}\right\}_{\xi \in \Gamma}$ in $\mathcal{H}$ we have

$$
\sum_{\xi, \eta \in \Gamma}\left[T_{\xi^{*} \eta} g_{\eta}, g_{\xi}\right] \geq 0 .
$$

(c) For any $\alpha \in \Gamma$ there exists a nonnegative number $c(\alpha)$ such that for any finitely supported family $g=\left\{g_{\xi}\right\}_{\xi \in \Gamma}$ in $\mathcal{H}$ we have

$$
\sum_{\xi, \eta \in \Gamma}\left[T_{\xi^{*} \alpha^{*} \alpha \eta} g_{\eta}, g_{\xi}\right] \leq c(\alpha)^{2} \sum_{\xi, \eta \in \Gamma}\left[T_{\xi^{*} \eta} g_{\eta}, g_{\xi}\right] \text {. }
$$

(2) There exists a VH-space $\mathcal{K}, a *$-representation $D=\left\{D_{\xi}\right\}_{\xi \in \Gamma}$ of $\Gamma$ in $\mathcal{B}^{*}(\mathcal{K})$, and an operator $V \in \mathcal{B}^{*}(\mathcal{H}, \mathcal{K})$, such that

$$
T_{\xi}=V^{*} D_{\xi} V, \quad \xi \in \Gamma .
$$

Moreover, under the assumption of Theorem 5.2, the VH-space $\mathcal{K}$ can be obtained minimal in the sense that it is spanned by elements of the form $D_{\xi} V f$, where $f \in \mathcal{H}$ and $\xi \in \Gamma$ and, in this case, the triple $(\mathcal{K} ; D ; V)$ is uniquely determined up to an isomorphism of $\mathrm{VH}$-spaces that intertwines the $*$-representations and keeps the corresponding operators $V$.

In addition, in case $T_{\epsilon}=I, \mathcal{H}$ can be isometrically embedded as an orthocomplemented subspace into $\mathcal{K}$ and, letting $P_{\mathcal{H}}$ be the orthogonal projection onto $\mathcal{H}$, we have

$$
T_{\xi}=\left.P_{\mathcal{H}} D_{\xi}\right|_{\mathcal{H}}, \quad \xi \in \Gamma .
$$


As a consequence of the reproducing kernel version for invariant kernels, see Theorem 4.2.(3), and the reproducing kernel version of the linearization as in Proposition 5.1, we get the following:

Corollary 5.3. With the notation as in Theorem 5.2, each of the assertions (1) and (2) is equivalent with

(3) $T$ admits a reproducing kernel VH-space $\mathcal{R}$ over $Z$, in the sense of the properties (a)-(c) in Proposition 5.1(3), and a $*$-representation $\rho: \Gamma \rightarrow$ $\mathcal{B}^{*}(\mathcal{R})$, such that

(d) $\rho: \Gamma \rightarrow \mathcal{B}^{*}(\mathcal{R})$ is a $*$-representation such that $\rho(\xi) T_{\eta} . h=T_{\eta \xi^{*}} . h$, in the sense that map $\Gamma \ni \gamma \mapsto \rho(\xi) T_{\eta \gamma} h \in \mathcal{H}$ coincides with the map $\Gamma \ni \gamma \mapsto T_{\eta \xi^{*} \gamma} h \in \mathcal{H}$, for all $\xi, \eta \in \Gamma$ and all $h \in \mathcal{H}$.

Also, the pair $(\mathcal{R} ; \rho)$ is uniquely determined by $T$, with properties (a)-(d).

\subsection{Stinespring's Dilation Theorem in VH-Spaces}

Let $\mathcal{A}$ be a complex $*$-algebra with unit 1 . Recall that the involution $*$ is supposed to be conjugate linear, $(a b)^{*}=b^{*} a^{*}$ for all $a, b \in \mathcal{A},\left(a^{*}\right)^{*}=a$ for all $a \in \mathcal{A}$, and that $1^{*}=1$. In particular, $\mathcal{A}$ has an underlying structure of a unital multiplicative $*$-semigroup. Also, by definition, an element $a \in \mathcal{A}$ is positive if $a=x^{*} x$ for some $x \in \mathcal{A}$. This definition, for general $*$-algebras, may not mean too much, but it is the right definition in the case of $C^{*}$-algebras.

For an arbitrary VH-space $\mathcal{H}$ over the admissible space $Z$, let $\varphi: \mathcal{A} \rightarrow$ $\mathcal{B}^{*}(\mathcal{H})$ be a linear map. A kernel $K: \mathcal{A} \times \mathcal{A} \rightarrow \mathcal{B}^{*}(\mathcal{H})$ can be defined by

$$
K(b, a)=\varphi\left(a^{*} b\right), \quad a, b \in \mathcal{A} .
$$

With this observation, the types of positivity for kernels, as in Sects. 3.1 and 5.1, have transcriptions to this setting: $\varphi$ is n-positive, for some natural number $n$, if for any $a_{1}, \ldots, a_{n} \in \mathcal{A}$ and $h_{1}, \ldots, h_{n} \in \mathcal{H}$ we have

$$
\sum_{i, j=1}^{n}\left[\varphi\left(a_{i}^{*} a_{j}\right) h_{j}, h_{i}\right]_{\mathcal{H}} \geq 0,
$$

and, respectively, $\varphi$ is positive semidefinite if it is $n$-positive for all $n \in \mathbb{N}$.

There is another notion of positivity that has been considered, following the original terminology of Stinespring [29]. Given $\mathcal{A}$ a *-algebra, a linear map $\varphi: \mathcal{A} \rightarrow \mathcal{B}^{*}(\mathcal{H})$ is called positive if $\varphi\left(a^{*} a\right) \geq 0$ for any $a \in \mathcal{A}$. Given $n \in \mathbb{N}$, there is a natural identification of $*$-algebras of $M_{n}(\mathcal{A})$, the algebra of all $n \times n$ matrices with entries in $\mathcal{A}$, with $M_{n} \otimes \mathcal{A}$, organized as a *-algebra similarly in a natural way (e.g. see [27]). A linear map $\varphi_{n}: M_{n}(\mathcal{A}) \rightarrow M_{n}\left(\mathcal{B}^{*}(\mathcal{H})\right)$ is naturally associated to $\varphi$ by

$$
\varphi_{n}\left(\left[a_{i, j}\right]_{i, j=1}^{n}\right)=\left[\varphi\left(a_{i, j}\right)\right]_{i, j=1}^{n}, \quad\left[a_{i, j}\right]_{i, j=1}^{n} \in M_{n}(\mathcal{A}) .
$$

The importance of this construction, and its consequences in terms of positivity, relies on its "quantization" interpretation, which basically comes from the interpretation of the tensor product of two Hilbert spaces as the aggregate of two quantum systems. 
Taking into account that any positive element $A=\left[a_{i, j}\right]_{i, j=1}^{n} \in M_{n}(\mathcal{A})$ can be decomposed

$$
A=A_{1}^{*} A_{1}+\cdots+A_{n}^{*} A_{n},
$$

where $A_{k}$ is the $n \times n$ matrix having its $k$-th row with entries $a_{k, j}, j=1, \ldots, n$, and all the other entries null, we get the following fact, essentially proven in [29]:

Proposition 5.4. Let $\mathcal{A}$ be a *-algebra, $\mathcal{H}$ a VH-space, and a linear map $\varphi: \mathcal{A} \rightarrow \mathcal{B}^{*}(\mathcal{H})$.

(a) For arbitrary $n \in \mathbb{N}, \varphi$ is n-positive if and only if $\varphi_{n}$ is positive.

(b) $\varphi$ is positive semidefinite if and only if $\varphi_{n}$ is positive for all $n \in \mathbb{N}$.

$\varphi$ is called completely positive if $\varphi_{n}$ is positive for all $n \in \mathbb{N}$, hence, Proposition 5.4 says that complete positivity is the same with positive semidefiniteness, in this setting.

We make now the observation that the kernel $K$ defined as in (5.7) is invariant under the action of $\mathcal{A}$ on itself by left multiplication, more precisely,

$$
K(b, c \cdot a)=\varphi\left((c a)^{*} b\right)=\varphi\left(a^{*} c^{*} b\right)=K\left(c^{*} \cdot b, a\right), \quad a, b, c \in \mathcal{A} .
$$

Thus, dilations of completely positive maps $\varphi$ fall under Theorem 4.2 for dilations of positive semidefinite kernels that are invariant under actions of *-semigroup. However, there is an important difference due to the fact that the rich structure of the $B^{*}$-algebra yields the boundedness condition (4.4) for free, in this special setting; we briefly recall the argument in [11]. Let $a \in \mathcal{A}$ and finitely supported $\left\{h_{b}\right\}_{b \in \mathcal{A}}$ in $\mathcal{H}$. Since $\varphi$ is positive semidefinite, for any $y \in \mathcal{A}$ we have

$$
\sum_{b, c \in \mathcal{A}}\left[\varphi\left(c^{*} y^{*} y b\right) h_{b}, h_{c}\right]_{\mathcal{H}} \geq 0 .
$$

Without loss of generality we can assume that $\|a\|<1$ and let $x=a^{*} a$, hence $\|x\| \leq\|a\|^{2}<1$. Following an idea in [3] and using an exercise in [2] at page 125 , we consider the power series of the analytic complex function $(1-\lambda)^{1 / 2}$ that converges in the open unit disc

$$
(1-\lambda)^{1 / 2}=1-\sum_{n \geq 1} c_{n} \lambda^{n}
$$

and let

$$
y=1-\sum_{n \geq 1} c_{n} x^{n} \in \mathcal{A} .
$$

It is easy to see that $y=y^{*}$, since $x=x^{*}$, and that $1-a^{*} a=1-x=y^{2}$, hence, from (5.10) it follows

$$
\sum_{b, c \in \mathcal{A}}\left[\varphi\left(c^{*} a^{*} a b\right) h_{b}, h_{c}\right]_{\mathcal{H}} \leq \sum_{b, c \in \mathcal{A}}\left[\varphi\left(c^{*} b\right) h_{b}, h_{c}\right]_{\mathcal{H}},
$$

which proves that, there exists a nonnegative number $c(a)$ such that

$$
\sum_{b, c \in \mathcal{A}}\left[\varphi\left(c^{*} a^{*} a b\right) h_{b}, h_{c}\right]_{\mathcal{H}} \leq c(a)^{2} \sum_{b, c \in \mathcal{A}}\left[\varphi\left(c^{*} b\right) h_{b}, h_{c}\right]_{\mathcal{H}} .
$$


The following theorem summarizes the consideration from above in the form of a Stinespring type theorem that falls under Theorem 4.2. In [11] this theorem has been proven in two other different ways.

Theorem 5.5. Let $\mathcal{A}$ be a unital $B^{*}$-algebra, $\mathcal{H}$ a VH-space over an admissible space $Z$, and let $\varphi: \mathcal{A} \rightarrow \mathcal{B}^{*}(\mathcal{H})$ be a linear map. Then $\varphi$ is a completely positive if and only if there exists $\mathcal{K}$ a $V H$-space over the same admissible space $Z$, an operator $V \in \mathcal{B}^{*}(\mathcal{H}, \mathcal{K})$ and a*-representation $\rho: \mathcal{A} \rightarrow \mathcal{B}^{*}(\mathcal{K})$ such that

$$
\varphi(a)=V^{*} \rho(a) V, \text { for all } a \in \mathcal{A} .
$$

Moreover, the $V H$-space $\mathcal{K}$ can be obtained minimal in the sense that $\mathcal{K}=\overline{\operatorname{Lin}\{\varphi(\mathcal{A}) \mathcal{H}\}}$ and, in this case, the triple $(\rho ; V ; \mathcal{K})$ is unique, modulo a unitary operator of $V H$-spaces that intertwines the $*$-representations and keeps the operators $V$.

In addition, if $\varphi$ is unital, $\mathcal{H}$ can be isometrically embedded as an orthocomplemented subspace of $\mathcal{K}$ and, letting $P_{\mathcal{H}}$ denote the orthogonal projection onto $\mathcal{H}$, we have

$$
\varphi(a)=\left.P_{\mathcal{H}} \rho(a)\right|_{\mathcal{H}}, \text { for all } a \in \mathcal{A} .
$$

Finally, we point out that, in the context of Theorem 5.5, a reproducing kernel representation can always be obtained, similarly as in Corollary 5.3.

Corollary 5.6. Let $\mathcal{A}$ be a unital $B^{*}$-algebra, $\mathcal{H}$ a VH-space over an admissible space $Z$, and $\varphi: \mathcal{A} \rightarrow \mathcal{B}^{*}(\mathcal{H})$ be a completely positive linear map. Then, there exists a pair $(\mathcal{R} ; \pi)$ subject to, and uniquely determined by, the following properties:

(i) $\mathcal{R}$ consists of functions $f: \mathcal{A} \rightarrow \mathcal{H}$ only.

(ii) $\varphi(a \cdot) h \in \mathcal{R}$, that is, the function $\mathcal{A} \ni b \mapsto \varphi(a b) h \in \mathcal{H}$ belongs to $\mathcal{R}$, for all $a \in \mathcal{A}$ and all $h \in \mathcal{H}$.

(iii) $[f(a), h]_{\mathcal{H}}=\left[f, \varphi\left(a^{*} \cdot\right) h\right]_{\mathcal{R}}$ for all $f \in \mathcal{R}, a \in \mathcal{A}$, and $h \in \mathcal{H}$.

(iv) $\pi: \mathcal{A} \rightarrow \mathcal{B}^{*}(\mathcal{R})$ is a *-algebra representation such that $\pi(a) \varphi(b \cdot) h=$ $\varphi\left(b a^{*}.\right) h$ for all $a, b \in \mathcal{A}$ and all $h \in \mathcal{H}$.

\section{References}

[1] Aronszajn, N.: Theory of reproducing kernels. Trans. Am. Math. Soc. 68, 337$404(1950)$

[2] Arveson, W.B.: A Short Course on Spectral Theory. Graduate Texts in Mathematics, vol. 209. Springer, Berlin-Heidelberg-New York (2002)

[3] Arveson, W.B.: Dilation theory yesterday and today. In: A Glimpse at Hilbert Space Operators. pp. 99-123. Oper. Theory Adv. Appl., vol. 207. Birkhäuser Verlag, Basel (2010)

[4] Constantinescu, T., Gheondea, A.: Representations of Hermitian kernels by means of Krein spaces. Publ. RIMS. Kyoto Univ. 33, 917-951 (1997)

[5] Constantinescu, T., Gheondea, A.: Representations of Hermitian kernels by means of Krein spaces. II. Invariant kernels. Commun. Math. Phys. 216, 409$430(2001)$ 
[6] Constantinescu, T., Gheondea, A.: On L. Schwartz's boundedness condition for kernels. Positivity 10, 65-86 (2006)

[7] Evans, D.E., Lewis, J.T.: Dilations of Irreducible Evolutions in Algebraic Quantum Theory. Comm. Dublin Inst. Adv. Studies Ser. A No. 24. Dublin Institute for Advanced Studies, Dublin (1977)

[8] Gaşpar, D., Gaşpar, P.: An operatorial model for Hilbert $\mathcal{B}(X)$-modules. Analele Univ. de Vest Timişoara. Ser. Mat.-Inform. 40, 15-29 (2002)

[9] Gaşpar, D., Gaşpar, P.: Reproducing kernel Hilbert $B(X)$-modules. An. Univ. Vest Timişoara Ser. Mat.-Inform. 43(2), 47-71 (2005)

[10] Gaşpar, D., Gaşpar, P.: Reproducing kernel Hilbert modules over locally $C^{*}$ algebras. An. Univ. Vest Timişoara Ser. Mat.-Inform. 45(1), 245-252 (2007)

[11] Gheondea, A., Ugurcan, B.E.: On two equivalent dilation theorems in VH-spaces. Complex Anal. Operator Theory 6, 625-650 (2012)

[12] Heo, J.: Reproducing kernel Hilbert $C^{*}$-modules and kernels associated to cocycles. J. Math. Phys. 49, 103507 (2008)

[13] Inoue, A.: Locally $C^{*}$-algebras. Mem. Fac. Sci. Kyushu Univ. Ser. A 25, 197235 (1971)

[14] Kasparov, G.G.: Hilbert $C^{*}$-modules: theorems of Stinespring and Voiculescu. J. Operator Theory 4, 133-150 (1980)

[15] Kolmogorov, A.N.: Stationary sequences in Hilbert space. Bull. Math. Univ. Moscow 2, 1-40 (1941)

[16] Lance, E.C.: Hilbert $C^{*}$-Modules. A toolkit for operator algebraists. London Mathematical Society Lecture Note Series, vol. 210. Cambridge University Press, Cambridge (1995)

[17] Loynes, R.M.: On generalized positive-definite function. Proc. London Math. Soc. III. Ser. 15, 373-384 (1965)

[18] Loynes, R.M.: Linear operators in $V H$-spaces. Trans. Am. Math. Soc. 116, $167-$ 180 (1965)

[19] Loynes R.M.: Some problems arising from spectral analysis. In: Symposium on Probability Methods in Analysis (Loutraki, 1966), pp. 197-207. Springer, Berlin (1967)

[20] Manuilov, V., Troitsky, E.: Hilbert $C^{*}$-Modules. American Mathematical Society, Providence (2005)

[21] Mercer, J.: Functions of positive and negative type and their connection with the theory of integral equations. Philos. Trans. Roy. Soc. Lond. Ser. A 209, 415446 (1909)

[22] Moore, E.H.: General Analysis. Mem. Amer. Philos. Soc., Part I, Philadelphia 1935; Part II, Philadelphia (1939)

[23] Murphy, G.J.: Positive definite kernels and Hilbert $C^{*}$-modules. Proc. Edinburgh Math. Soc. 40, 367-374 (1997)

[24] Naŭmark, M.A.: On the representations of additive operator set functions. C.R. (Doklady) Acad. Sci. USSR 41, 359-361 (1943)

[25] Naŭmark, M.A.: Positive definite operator functions on a commutative group. Bull. (Izv.) Acad. Sci. USSR 7, 237-244 (1943)

[26] Parthasaraty, K.R., Schmidt, K.: Positive-Definite Kernels, Continuous Tensor Products and Central Limit Theorems of Probability Theory. Lecture Notes in Mathematics, vol. 272. Springer, Berlin (1972) 
[27] Paulsen, V.R.: Completely Bounded Maps and Operator Algebras. Cambridge University Press, Cambridge (2002)

[28] Phillips, N.C.: Inverse limits of $C^{*}$-algebras. J. Operator Theory 19, 159195 (1988)

[29] Stinespring, W.F.: Positive functions on $C^{*}$-algebras. Proc. Am. Math. Soc. 6, 211-216 (1955)

[30] Szafraniec, F.H.: Dilations of linear and nonlinear maps. In: Functions, Series, Operators, vols. I, II (Budapest, 1980), pp. 1165-1169. Colloq. Math. Soc. Janos Bolyai, 35. North-Holland, Amsterdam (1983)

[31] Sz.-Nagy B.: Prolongement des transformations de l'espace de Hilbert qui sortent de cet espace. In: Appendice au livre "Leçons d'analyse fonctionnelle" par F. Riesz et B. B. Sz.-Nagy, pp. 439-573. Akademiai Kiado, Budapest (1955)

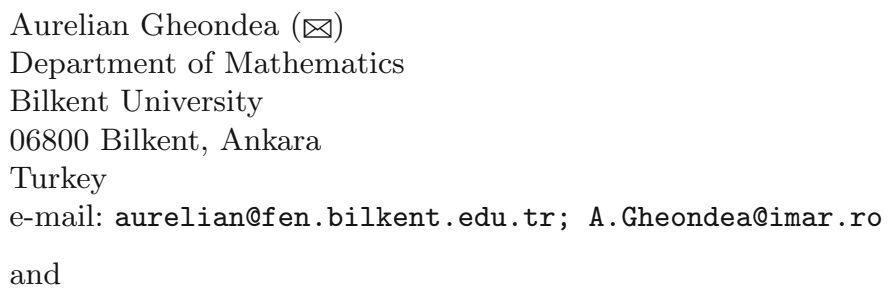

Received: March 13, 2012.

Revised: October 11, 2012. 